\title{
Highlights of Discoveries for $\delta$ Scuti Variable Stars From the Kepler Era
}

\author{
Joyce Ann Guzik* \\ Los Alamos National Laboratory, Los Alamos, NM, United States
}

The NASA Kepler and follow-on K2 mission (2009-2018) left a legacy of data and discoveries, finding thousands of exoplanets, and also obtaining high-precision long time-series data for hundreds of thousands of stars, including many types of pulsating variables. Here we highlight a few of the ongoing discoveries from Kepler data on $\delta$ Scuti pulsating variables, which are core hydrogen-burning stars of about twice the mass of the Sun. We discuss many unsolved problems surrounding the properties of the variability in these stars, and the progress enabled by Kepler data in using pulsations to infer their interior structure, a field of research known as asteroseismology.

Keywords: stars: $\delta$ Scuti, stars: $\gamma$ Doradus, NASA Kepler mission, asteroseismology, stellar pulsation

\section{OPEN ACCESS}

Edited by:

Karen Kinemuchi,

New Mexico State University,

United States

Reviewed by:

Dominic Bowman,

KU Leuven, Belgium

Mike Reed,

Missouri State University

United States

*Correspondence:

Joyce Ann Guzik

joy@lanl.gov

Specialty section:

This article was submitted to

Stellar and Solar Physics,

a section of the journal

Frontiers in Astronomy and Space

Sciences

Received: 14 January 2021

Accepted: 22 March 2021

Published: 20 April 2021

Citation:

Guzik JA (2021) Highlights of Discoveries for $\delta$ Scuti Variable Stars

From the Kepler Era.

Front. Astron. Space Sci. 8:653558. doi: 10.3389/fspas.2021.653558

\section{INTRODUCTION}

The long time-series, high-cadence, high-precision photometric observations of the NASA Kepler (2009-2013) (Borucki et al., 2010; Gilliland et al., 2010; Koch et al., 2010) and follow-on K2 (2014-2018) (Howell et al., 2014) missions have revolutionized the study of stellar variability. The amount and quality of data provided by Kepler is nearly overwhelming, and will motivate follow-on observations and generate new discoveries for decades to come.

Here we review some highlights of discoveries for $\delta$ Scuti (abbreviated as $\delta$ Sct) variable stars from the Kepler mission. The $\delta$ Sct variables are pre-main-sequence, main-sequence (core hydrogen-burning), or post-main-sequence (undergoing core contraction after core hydrogenburning, and beginning shell hydrogen-burning) stars with spectral types A through mid-F, and masses around two solar masses. They pulsate in one or more radial and non-radial modes with periods of around $2 \mathrm{~h}$. The pulsations are driven mainly by the " $\kappa$-effect" (opacity-valving) mechanism in the region of the second ionization of helium at temperatures around $50,000 \mathrm{~K}$ in the stellar envelope (Aerts et al., 2010).

Several reviews on Kepler findings for $\delta$ Sct variables have already been written (see, e.g., Balona, 2018; Bowman and Kurtz, 2018), and a comprehensive review of the Kepler legacy for these stars is premature. Prior to Kepler, one of the best compilations of the state-of-the-art of research on $\delta$ Sct variables was the Handbook and Conference Proceedings volume $\delta$ Scuti and Related Stars (Breger and Montgomery, 2000). New catalogs and lists of variable stars, including $\delta$ Sct stars observed and first discovered by the Kepler/K2 missions, have begun to appear [e.g., Bradley et al., 2015, 2016, $84 \delta$ Sct and 32 hybrid (see section 2.1) candidates from Kepler Guest Observer Program Cycle 1-5 observations; Murphy et al., 2019, $1988 \delta$ Sct stars from Kepler observations; Guzik et al., 2019, 249 $\delta$ Sct candidates from $\mathrm{K} 2$ observations].

After the successes of asteroseismology to infer the interior structure of the Sun and properties of sun-like stars, studying the slightly more massive $\delta$ Sct stars appeared to be a promising next direction for asteroseismology. Before the space observations of Kepler, CoRoT (see, e.g., Poretti et al., 2009), and MOST (see, e.g., Matthews, 2007), there existed only around a dozen $\delta$ Sct stars 
with long time-series observations from ground-based networks allowing the detection of a large number of pulsation modes [e.g., FG Vir (Breger et al., 2005) or 4 CVn (Breger et al., 2017)] that could be used to constrain stellar models. The field of $\delta$ Sct asteroseismology has been impeded by the problem of mode identification for several reasons. Unlike for the Sun, the disks of distant stars cannot be highly resolved, so only low-degree $(\ell \lesssim 3)$ mode variations that do not average out over the disk can be detected photometrically. Furthermore, most of these stars rotate more rapidly than the Sun, resulting in large and uneven rotational splittings, such that multiplets of adjacent modes can overlap. In addition, not all of the modes expected by non-adiabatic pulsation calculations are found in the observations. Finally, the modes are of low radial order $n$, and therefore the spacing pattern is not expected to show regular large separations seen for the higher-order stochastically excited solar-like modes where $n \sim 20$ and the modes can be described using asymptotic theory $(n \gg \ell$; see, e.g., García and Ballot, 2019). Unlike for the Sun, fundamental properties of a single $\delta$ Sct variable (mass, radius, age, detailed element abundances) cannot be derived from complementary or independent observations (e.g., meteorites, Earth, or planetary orbits). $\delta$ Sct stars in clusters, binaries, or having planetary systems are therefore useful to provide additional constraints for modeling.

The A-F main-sequence stars occupy a small region in the center of the H-R diagram, but have been differentiated into not only $\delta$ Sct and $\gamma$ Doradus (abbreviated as $\gamma$ Dor) variables (Pollard, 2009; Balona et al., 2011a), but also metallic-line A (Am) (Smalley et al., 2017), peculiar abundance A (Ap) (Mathys et al., 2020), rapidly-oscillating Ap (roAp) (Holdsworth, 2021) stars, $\alpha^{2}$ CVn variables (Sikora et al., 2019), $\lambda$ Boo stars (Murphy and Paunzen, 2017), the High Amplitude $\delta$ Sct (HADS) (McNamara, 2000) and SX Phe (Nemec et al., 2017) stars, blue stragglers (Rain et al., 2021), and the maybe "mythical"1 Maia variables (Breger, 1980; Cox, 1983; Daszyńska-Daszkiewicz et al., 2017; White et al., 2017; Balona, 2018). Kepler observations have revealed overlap and commonalities among these types, pointing the way to a more fundamental understanding of the origins of the diverse phenomena seen in these stars.

While these stars were expected at first to be the next straightforward step beyond the solar-like oscillators for applications of asteroseismology, this goal has turned out to be more difficult to achieve than expected. However, the many complexities of these stars make this field of variable star research rich in potential discoveries.

\footnotetext{
${ }^{1}$ This term was coined by Breger (1980) re. reports of pulsating variables between the main-sequence $\delta$ Sct and Slowly-Pulsating B-type stars in the H-R diagram. There has been debate in the literature about whether these variables actually exist, what drives their pulsations, and whether they should be considered a separate class of variable stars. The prototype Maia, a member of the Pleiades, has been shown not to pulsate (see, e.g., White et al., 2017). Other proposed members of the class may be rapidly rotating SPB stars, or be slowly rotating, but have pulsations driven by the $\kappa$ effect with enhanced opacity around $125,000 \mathrm{~K}$ (Daszyńska-Daszkiewicz et al., 2017).
}

\section{DISCOVERY HIGHLIGHTS}

\subsection{Pre- and Post-Kepler View-Hybrids Everywhere!}

Before Kepler, the $\delta$ Sct and $\gamma$ Dor stars and their hybrids were found in the instability regions expected by theory. The $\delta$ Sct $p$-mode pulsations are driven by the $\kappa$ mechanism in their radiative envelopes, but the longer period (1-3 day) $\gamma$ Dor $g$ mode pulsations are proposed to be driven by the "convective blocking" mechanism operating at the base of their convective envelope around 300,000 K (Guzik et al., 2000). Using a timedependent convection treatment, hybrid stars pulsating in both $p$ and $g$ modes were expected and found in a small region of the H-R diagram where these two instability regions overlapped (Dupret et al., 2005).

Just after the first Kepler light curves were received, it became apparent that this picture would be shattered. $\delta$ Sct and $\gamma$ Dor variables and their hybrids were found throughout and even somewhat beyond the edges of the combined instability regions (Grigahcène et al., 2010; Uytterhoeven et al., 2011) (see Figure 1). Low-frequency pulsation modes identified using the long (30min) cadence Kepler data could have been mis-identified as $\gamma$ Dor $g$ modes, but actually may be Nyquist reflections of frequencies above $24.5 \mathrm{~d}^{-1}$. However, the low frequencies are also found using short (1-min) cadence data, and Nyquist reflection frequencies can be distinguished using a long-enough series (near one Kepler orbital period of 375.2 d) of long-cadence data (Murphy et al., 2013). Some low frequencies could possibly be caused by rotation/starspots, undetected binary companions, rotational perturbations of higher frequency modes, combination frequencies, a background star or nearby bright star in the field of view contaminating the light curve, or Rossby or Kelvin waves. However, Balona (2014), using only short-cadence data, ruled out most of these explanations, and arrives at the bold conclusion that "all $\delta$ Sct stars are essentially $\delta$ Sct $/ \gamma$ Dor hybrids."

On the other hand, there exist examples of $\delta$ Sct stars that do not show $g$-mode pulsations in the Kepler data. Bowman (2017) comments on Balona's claim, and discusses an example of a "pure" $\delta$ Sct star KIC 5617488, which has no low-frequency peaks with $S / N \geq 4$. The few low-frequency peaks visible in the amplitude spectrum have amplitude less a few $\mu$ mag. It is possible that $g$ modes with angular degree $\ell \gtrsim 3$ are undetected in many stars photometrically, but may be discovered spectroscopically. Such modes have been identified in $\gamma$ Dor variables (Pollard et al., 2013), but usually are also accompanied by higher amplitude $\ell=$ 1 modes.

New pulsation driving mechanisms are being investigated. For example, Balona et al. (2015b) find that an opacity increase of about a factor of two near temperatures of 115,000 K $(\log T=$ $5.06)$ in the stellar envelope can result in instability of some lowfrequency modes, but this opacity bump also reduces the range of unstable high-frequency modes. Balona (2018) highlights theoretical and computational work by Xiong et al. (2016) with a new treatment of time-dependent convection that allows $\delta$ Sct stars to pulsate in low-frequency modes. 


\subsection{The "Superstar" and a New Pulsation Driving Mechanism}

A $\delta$ Sct star that attracted early excitement was HD 187547 (KIC 7548479), known as the "superstar," observed by Kepler in short cadence. This star shows not only the expected $\delta$ Sct pulsation modes, but also some additional modes of somewhat higher frequency superimposed (Figure 2). Antoci et al. (2011) suggested that convection was stochastically exciting these modes, despite the fact that $\delta$ Sct star models do not have large efficient envelope convection zones, making this star the first $\delta$ Sct/solar-like oscillator discovered. However, continued Kepler observations showed that the mode lifetimes were quite long, longer than 960 days, and may in fact be "coherent," i.e., not stochastically excited. Antoci et al. (2014) proposed a new pulsation driving mechanism for these higher-frequency modes, the 'turbulent pressure' mechanism, operating in the outer convective layers of these stars. They illustrated this mechanism using models including a time-dependent convection treatment applied to radial modes. This discovery was one of several from the Kepler data resulting in a suggested new pulsation driving mechanism.

\section{3. "Constant" Stars in the $\delta$ Sct Instability Region}

While the Kepler data confused the picture of the instability regions for $\delta$ Sct and $\gamma$ Dor stars and their hybrids, these data
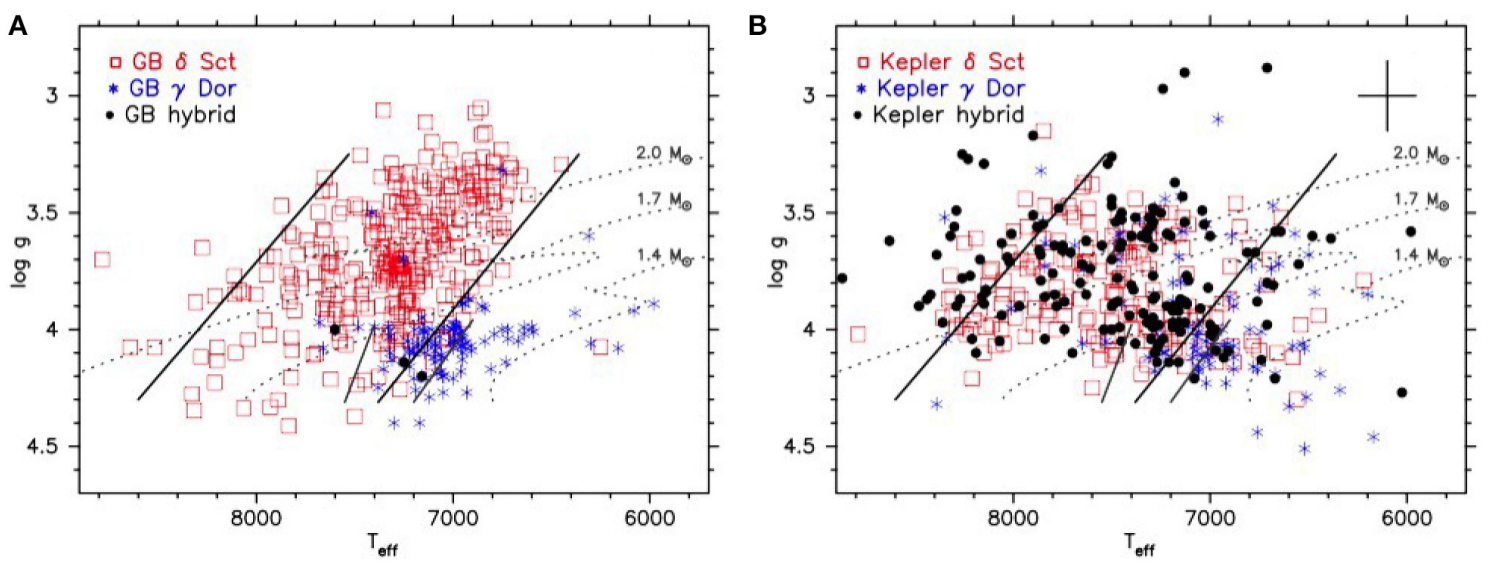

FIGURE 1 | Figure 10 from Uytterhoeven et al. (2011): (A) Log surface gravity vs. $T_{\text {eff }}$ for the $\delta$ Sct, $\gamma$ Dor, and hybrid stars detected from the ground (parameters taken from the literature). (B) Log surface gravity vs. $T_{\text {eff }}$ for Kepler stars classified as $\delta$ Sct, $\gamma$ Dor, and hybrid stars by Uytterhoeven et al. (2011). Open red squares represent $\delta$ Sct stars, blue asterisks indicate $\gamma$ Dor stars, and hybrid stars are marked by black bullets. The black cross in the right top corner shows typical errors on the values. Evolutionary tracks for main-sequence stars with masses 1.4, 1.7, and $2.0 \mathrm{M}_{\odot}$ are plotted with gray dotted lines. The solid thick black and light gray lines mark the blue and red edge of the observed instability strips of $\delta$ Sct and $\gamma$ Dor stars, as described by Rodríguez and Breger (2001) and Handler and Shobbrook (2002), respectively. Reproduced with permission @ ESO.
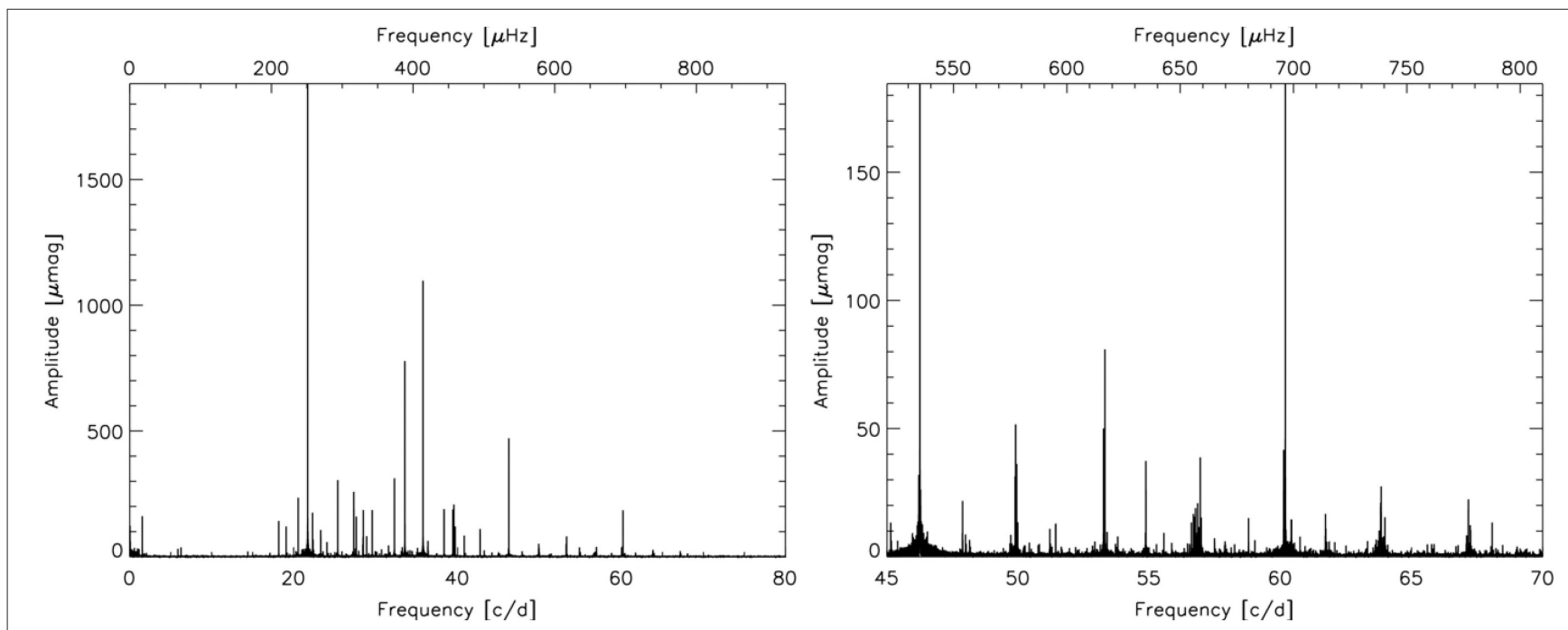

FIGURE 2 | Figure 1 from Antoci et al. (2014). (Left) Fourier spectra of the Kepler short-cadence data. (Right) Close-up in the frequency region interpreted by Antoci et al. (2011) to be stochastically excited. C AAS. Reproduced with permission. 
also affirmed that many of the stars in the $\delta$ Sct instability region of the H-R diagram are "constant," i.e., not pulsating, at least not at levels detectable by Kepler (Guzik et al., 2013, 2014, 2015a,b; Balona et al., 2015b; Murphy et al., 2015).

Balona et al. (2015b) found that 1,165 out of 2,839 stars (41\%) in the $\delta$ Sct temperature region are not pulsating according to Kepler photometry. Murphy et al. (2019) use Gaia DR2 (Gaia Collaboration et al., 2018) data to derive luminosities and to investigate the pulsator fraction in the instability strip as a function of effective temperature and luminosity, finding that the pulsator fraction peaks at around $70 \%$ in the middle of the instability strip.

Guzik et al. (2013, 2014, 2015a,b) studied two collections (633 and 2,100+ stars, respectively) of mostly faint stars in the original Kepler field, using long-cadence observations requested to search for $\delta$ Sct and $\gamma$ Dor candidates. They find many constant stars, showing no variability at the $20 \mathrm{ppm}$ level for frequencies between 0.2 and $24.5 \mathrm{~d}^{-1}$. Most are outside the $\gamma$ Dor and $\delta$ Sct instability regions, but they find six stars in their sample for Quarters 6-13 (Guzik et al., 2013, 2014), and 1552 stars, depending on the uncertainty and systematic errors adopted for the Kepler Input Catalog effective temperature and surface gravity, for Quarters 14-17 (Guzik et al., 2015a,b) that lie within the pulsation instability regions (see Figure 3 ).

Murphy et al. (2015) use high-resolution spectroscopy to investigate constant stars (defined as showing no $\delta$ Sct $p$ mode variations above $50 \mu \mathrm{mag}$ amplitude) within the $\delta$ Sct instability strip. They find that most of these stars have peculiar element abundances with enhancements and deficiencies of certain elements compared to solar abundances, and are classified as metallic-line A (Am) stars. The diffusive settling and radiative levitation believed to cause the abundance anomalies in Am stars would also be expected to deplete helium from the $\delta$ Sct pulsation driving region, and could explain why these stars are not pulsating. Setting aside the chemically-peculiar stars, Murphy et al. (2015) find that the remaining stars not pulsating in $\delta$ Sct $p$ modes are near the edges of the instability regions (Figure 4). Murphy et al. (2015) propose that some of these stars may be in undetected binaries, and therefore have inaccurate effective temperatures and actually may lie outside the instability strip; it is also possible that a binary companion could inhibit pulsations. Additional investigation is needed to determine whether these explanations apply for all of the "constant" stars.

\subsection{Spots and Flares}

Balona $(2012,2013,2015,2017,2019)$ found that around $40 \%$ of A-type stars observed by Kepler, including many $\delta$ Sct stars, show modulations in their light curves attributed to magnetic activity and starspots, and $1.5 \%$ even show flares. This behavior is surprising, because hotter stars, including Atype stars, are believed to have thin and inefficient envelope convection layers instead of a larger convective envelope as found in the Sun and cooler stars, and so are not expected to have convection+differential-rotation driven dynamos and magnetic cycles as found in solar-like stars. Balona (2019) published a paper titled "Evidence for spots on hot stars suggests major revision of stellar physics," conveying the significance of these

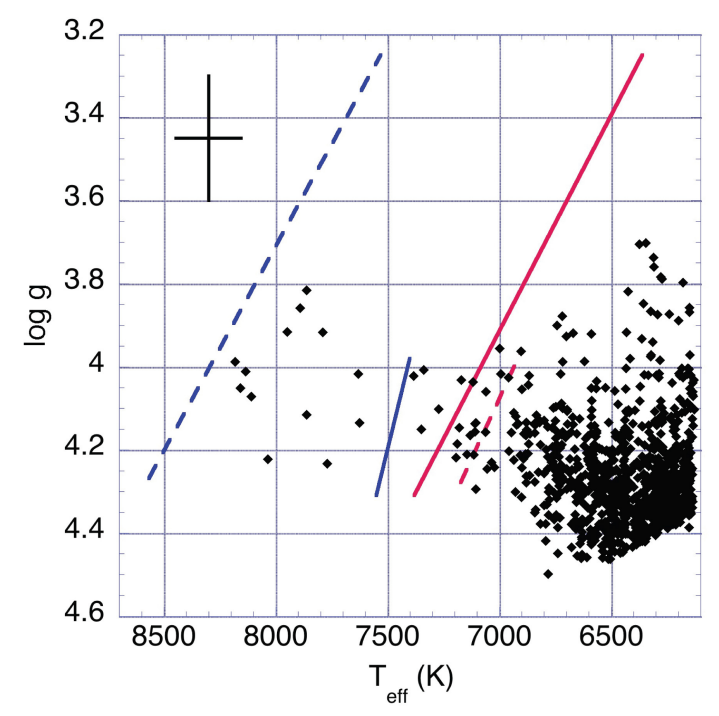

FIGURE 3 | Figure 1 from Guzik et al. (2015b): Location of stars that are "constant" in the log surface gravity- $T_{\text {eff }}$ diagram, along with $\delta$ Sct (dashed lines) and $\gamma$ Dor (solid lines) instability strip boundaries established from preKepler ground-based observations (Rodríguez and Breger, 2001; Handler and Shobbrook, 2002). The $T_{\text {eff }}$ of the sample stars has been shifted by $+229 \mathrm{~K}$ to account for the systematic offset between $T_{\text {eff }}$ of the Kepler Input Catalog and SDSS photometry for this temperature range as determined by Pinsonneault et al. (2012, 2013). The black cross shows an error bar on log g (0.3 dex) and $T_{\text {eff }}(290 \mathrm{~K})$ established by comparisons of $\mathrm{KIC}$ values and values derived from ground-based spectroscopy for brighter Kepler targets (Uytterhoeven et al., 2011). In this figure, 34 "constant" stars lie within the instability strip boundaries. Without the $+229 \mathrm{~K}$ offset, 17 "constant" stars would fall within the instability strip boundaries.

findings. It is possible that these stars retained a fossil field from their formation. It is also possible that a dynamo mechanism is operating in the convective core, if a way can be found for the field to diffuse through the overlying radiative layers quickly enough to reach the stellar surface (Brun et al., 2005; Featherstone et al., 2009).

Further investigations into A-type flaring stars have been conducted by Pedersen et al. (2017). They performed new analyses of the photometry of 33 flaring A-type stars listed by Balona (2012, 2013), verifying flares in 27 of these objects. In fourteen cases, an overlapping object in the Kepler pixel data may be responsible for the flares; in five other cases, the light curves are contaminated by nearby objects in the field. They also obtained new high-resolution spectroscopic observations of 22 of these stars, finding that eleven are spectroscopic binary systems, so that an unresolved low-mass companion may actually be producing the flares. Therefore, they have found possible alternative explanations for all but nine of these stars, six of them without high-resolution spectroscopy, casting some doubt on the A-star flare hypothesis.

Concerning the A-type stars, Balona $(2013,2017)$ attributed a broad unresolved hump of peaks with a higher amplitude sharp peak at the higher frequency edge in Kepler amplitude spectra to multiple star spots with finite lifetimes and differential rotation. 


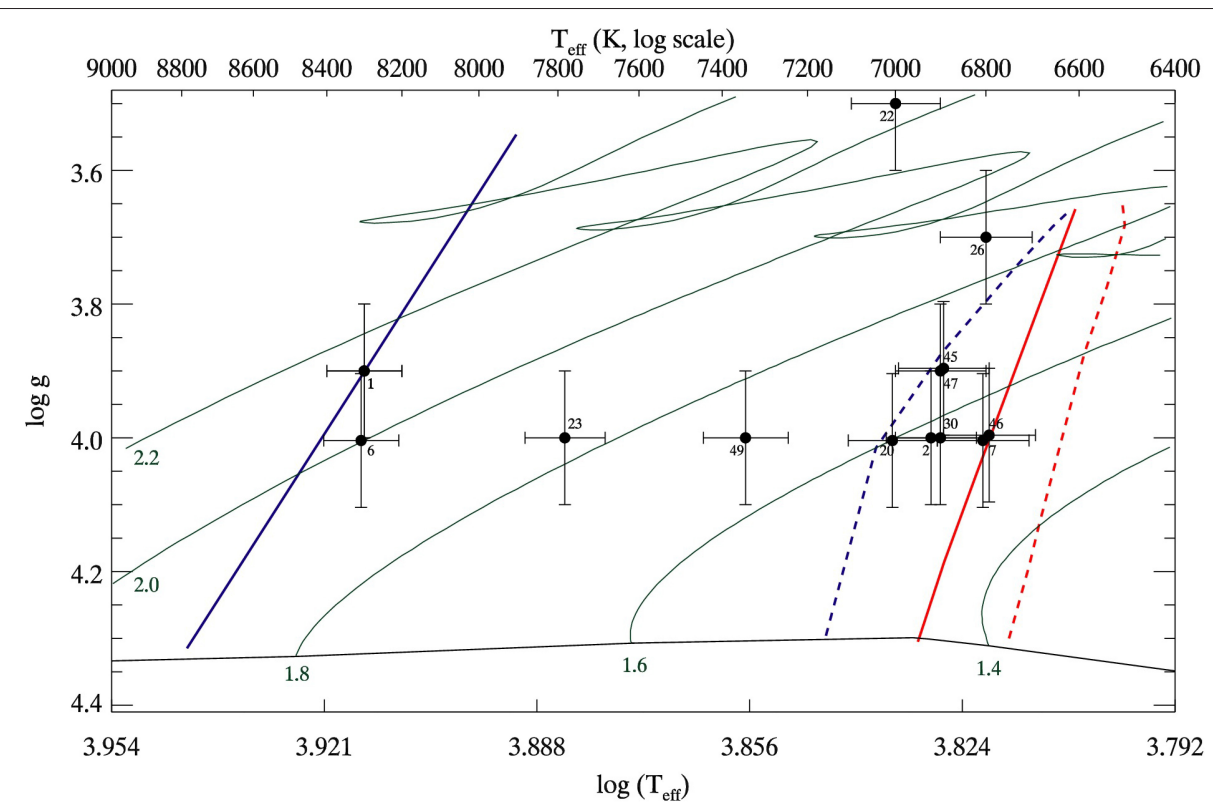

FIGURE 4 | Figure 2 from Murphy et al. (2015). Positions of chemically normal, non- $\delta$ Sct stars with $1 \sigma$ error bars. Solid blue and red lines are the blue and red edges of the $\delta$ Sct instability strip, while dashed lines indicate the $\gamma$ Dor instability strip. Green lines are evolutionary tracks, with masses in $\mathrm{M}_{\odot}$ written beneath the ZAMS (black). The non-pulsators generally lie near the edges of the $\delta$ Sct instability strip, with exceptions discussed in Murphy et al. (2015).

This broad hump has been explained by Saio et al. (2018) as $r$ modes (global Rossby waves). Saio et al. (2018) suggest that the resolved higher amplitude peak, usually accompanied by a few smaller peaks, is produced by one or a few long-lived star spots that could emerge in weakly magnetic A-type stars.

\subsection{Chemically Peculiar Stars}

The chemically peculiar A-type stars further challenge stellar pulsation and evolution theory. Diffusive settling of helium from the pulsation-driving region is expected to turn off the $\kappa$-effect mechanism and $\delta$ Sct pulsations in Am stars. However, some Am stars are observed to pulsate in $\delta$ Sct modes (Guzik et al., 2020; Murphy et al., 2020). Figure 5 from Murphy et al. (2020) shows the location of many Am stars including $\delta$ Sct pulsators, along with the blue edge of the $\delta$ Sct instability region calculated including diffusive settling of helium from the driving region. Murphy et al. (2020) find that pulsation driving from a Rosseland mean opacity bump at 50,000 K caused by the discontinuous $\mathrm{H}$ ionization edge in bound-free opacity explains the observation of $\delta$ Sct pulsations in Am stars. Smalley et al. (2017) propose that $\delta$ Sct pulsations in Am stars are driven by the turbulentpressure mechanism.

Balona et al. (2011b) find that the observed location of pulsating Am stars in the H-R diagram does not agree with the location predicted from diffusion calculations. Balona et al. (2015a) state: "The fact that so many Am stars are $\delta$ Sct variables is also at odds with the prediction of diffusion theory," and even suggest that accretion could be the origin of the metal enhancements.

Bowman et al. (2018) review magnetic chemically peculiar Atype (Ap) stars, including those that pulsate, observed during

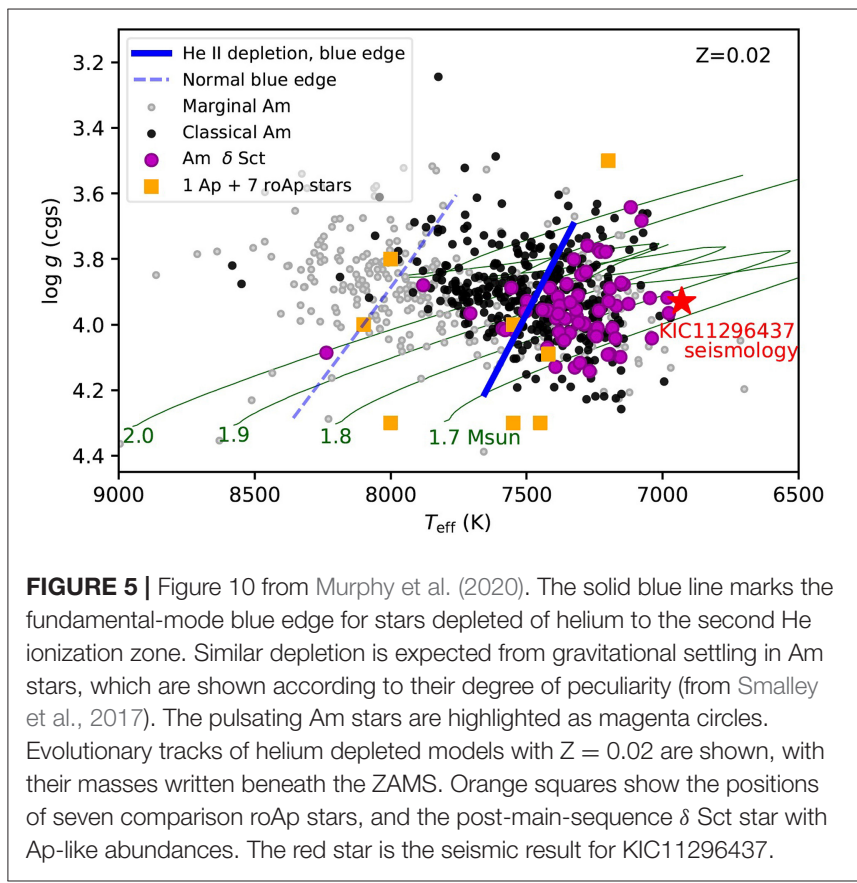

the Kepler K2 mission. Buysschaert et al. (2018) use spectropolarimetry to detect large-scale kilogauss magnetic fields in several chemically peculiar stars observed during the $\mathrm{K} 2$ mission. In Ap stars, "chemical spots" form at the magnetic poles that cause brightness contrasts that show up as light curve variations as the star rotates; these variables are also called $\alpha^{2}$ CVn variables. 
Misalignment of the dipole magnetic field axis and rotation axis is the preferred explanation for properties of high-frequency $p$ modes of the rapidly-oscillating Ap (roAp) stars (see Kurtz, 1982 and review by Holdsworth, 2021, this collection).

Strong magnetic fields, as are found in the Ap stars, are expected to suppress the low-overtone pulsations found in $\delta$ Sct stars. However, Murphy et al. (2020), using Kepler data, report the first $\delta$ Sct-roAp hybrid, KIC 11296437, having mean magnetic field modulus of $2.8 \pm 0.5$ kilogauss, and estimated polar magnetic field strength of 3.0-5.2 kilogauss. Figure 5 shows the location of this star on the H-R diagram based on asteroseismic models.

\subsection{HADS and SX Phe Stars}

Other A to mid-F spectral type pulsators are further divided into the high-amplitude $\delta$ Sct stars (HADS), and the related SX Phe stars. The Kepler data show that there may not be any physical distinction between SX Phe and HADS, or really between HADS and normal $\delta$ Sct stars.

The SX Phe stars are defined as Population II (low-metallicity) high-amplitude $\delta$ Sct stars, with one or two high-amplitude modes, and are usually found in globular clusters and in dwarf galaxies of the Local Group. They are bluer and brighter than the cluster turnoffs, and so are called "blue stragglers," which may have been formed by binary mergers. Balona and Nemec (2012) identified 34 blue straggler candidates in the original Kepler field based on their high tangential velocities (distance $x$ proper motion), which indicate that they belong to a thick disk or halo population (Figure 6). Nemec et al. (2017) supplemented the Kepler light curves with new spectroscopic observations to determine metallicity, temperatures, radial velocities, and projected rotational velocity $v \sin i$. They found that nearly all of these candidates had near-solar metallicities (Figure 7). Moreover, the Kepler light curves were not distinguishable from normal $\delta$ Sct stars, as they show complex spectra and even low frequencies as often seen in Kepler $\delta$ Sct light curves. It may turn out that the defining characteristic of field SX Phe stars, namely showing only one or two high-amplitude modes, is just a selection effect. There are also low-amplitude multi-periodic SX Phe stars found in globular clusters, lending support to the position that these stars should not be considered a separate class of pulsator from the normal $\delta$ Sct stars.

There is room for debate about whether SX Phe stars should be retained as a class of variables, and if so, how to define them and separate them from HADS or normal $\delta$ Sct stars. There are questions of cluster vs. field identification, metallicity (Pop. I or II), whether they have undergone mass transfer or merger, whether they are "blue stragglers," i.e., should have evolved off of the main sequence given their metallicity, age, and spectral type. It may be easiest to identify stars as SX Phe stars if they are found in globular clusters and are blue stragglers located on/near the main sequence above the turnoff. However, it is more difficult to distinguish them observationally if they are field stars. As discussed above, for the field stars, a high proper motion does not guarantee metallicity below solar, and so if one were to adopt the criteria of Pop. II metallicity and high proper motion, most of the Kepler SX Phe candidates would

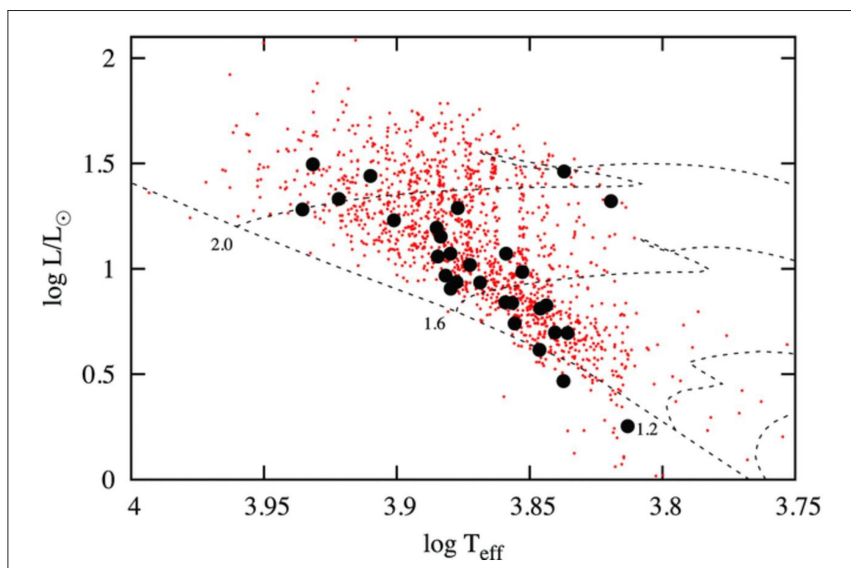

FIGURE 6 | Figure 2 from Balona and Nemec (2012). The theoretical H-R diagram for Kepler $\delta$ Sct stars (small filled circles). The large filled circles are $\delta$ Sct stars which have large proper motions and large tangential velocities (i.e., SX Phe candidates). Evolutionary tracks are shown and labeled with the solar mass.

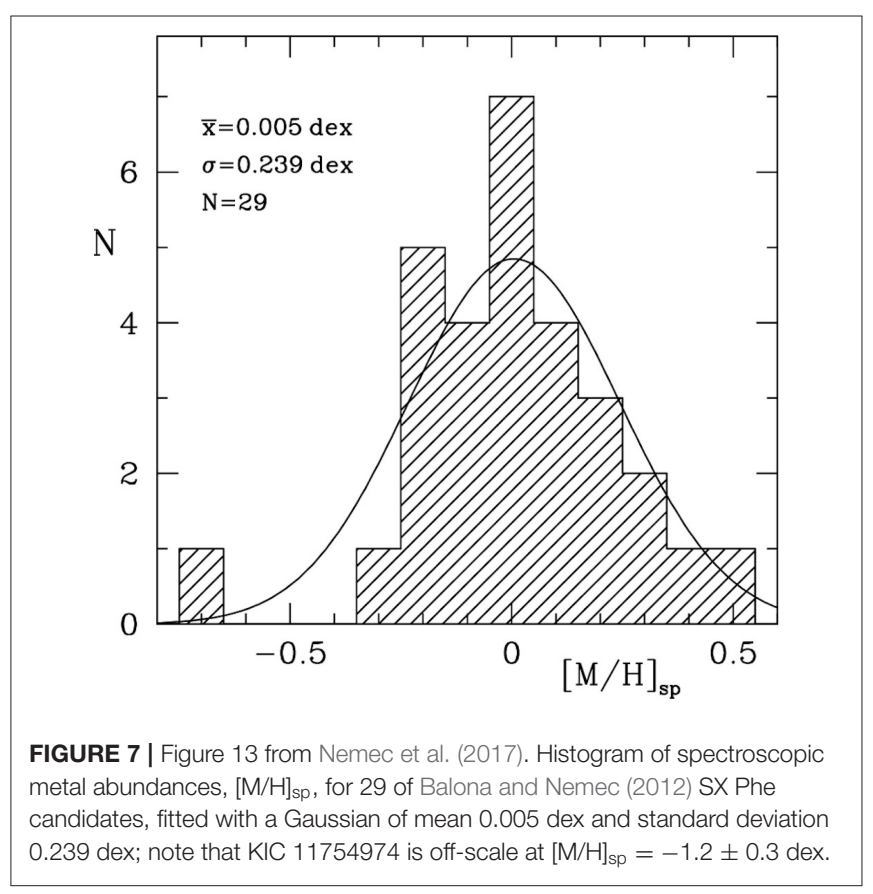

fail this test. Asteroseismic analysis of the prototype SX Phe (Daszyńska-Daszkiewicz et al., 2020a,b) shows that it is in the core contraction phase or shell H-burning phase, has age $\sim 4$ Gyr, and has a low mass $\left(\mathrm{M} \sim 1.05 \mathrm{M}_{\odot}\right)$ compared to HADS and $\delta$ Sct stars. The best-fit models have metallicity $\mathrm{Z}=0.0014-$ 0.002 , and favor a low hydrogen (high helium) abundance $\mathrm{X}=$ 0.67 (Daszyńska-Daszkiewicz et al., 2020b). One could consider adopting a mass plus age criterion to make the distinction, but these properties are not directly observable and would rely on asteroseismic analyses. Asteroseismic analyses fortunately show promise to constrain intrinsic metallicity and helium abundance, 
and possibly whether the star has experienced mass transfer from a binary companion or is the product of a binary merger, either of which could enhance the helium abundance. Should pulsation mode amplitude or the presence of only one or a few modes be retained as defining criteria? These questions will require further discussion.

\section{7. "Heartbeat" Stars and Tidally Excited Modes}

A new variable type categorized as a result of Kepler data are the "heartbeat" stars (Thompson et al., 2012; Fuller, 2017; Hambleton et al., 2018; Cheng et al., 2020; Guo et al., 2020), with KOI-54 (Welsh et al., 2011) being the first dramatic example observed by Kepler. These stars are binaries in highly eccentric $(\epsilon \gtrsim 0.3)$ orbits with orbital periods between 1 day and 1 year that show tidally induced oscillations (Fuller, 2017). These stars are sonamed because their light curves resemble an electrocardiogram, with a brightness dip followed immediately by a sharp rise at periastron. This feature is caused by increased tidal distortion of the components and viewing them at different angles at they orbit each other at periastron, and is also enhanced by light reflecting from the companion star and Doppler boosting at close approach. These binaries show tidally excited oscillations that can be identified because they are exact multiples of the binary orbital frequency. Some have one or more components that also show intrinsic pulsations, and some also show eclipses. Before Kepler, only a few such systems had been identified, but 17 systems were quickly discovered and characterized from Kepler data (Thompson et al., 2012), motivating their grouping as a new class of variables. It is interesting that Kirk et al. (2016b) catalog 176 heartbeat systems in the original Kepler field, but most have not been closely studied, and only around $20 \%$ actually show the expected tidally excited oscillations (Cheng et al., 2020).

One such Kepler eclipsing heartbeat star is KIC 4544587, studied by Hambleton et al. (2013), with eccentricity 0.28 , showing both high- and low-frequency modes typical of $\delta$ Sct and $\gamma$ Dor pulsations, as well as modes that are orbital frequency harmonics that may be excited by tidal resonances (See light curve excerpt in Figure 8). The masses of the two stars derived from binary modeling are 1.98 and $1.6 \mathrm{M}_{\odot}$.

An attempt was made to evolve models for each star with a common initial abundance and age, and using the same mixing length parameter, which best fit the constraints from binary orbits and pulsation modeling (Figure 9). Pulsation calculations for the stars matching the derived constraints point to the $1.6 \mathrm{M}_{\odot}$ star as most likely being the $\delta$ Sct pulsator. This exercise illustrated the potential of additional constraints from binaries to assist asteroseismic investigations.

\subsection{Amplitude Variations}

Most $\delta$ Sct asteroseismic studies have focused on periods and period spacings, but few have made use of the amplitudes of the pulsations. The study of amplitudes requires non-linear, nonradial, multidimensional hydrodynamic models which have not advanced far enough to predict mode selection and amplitudes of $\delta$ Sct stars. While the frequency content, amplitudes, and phases of some $\delta$ Sct stars have been documented to change with

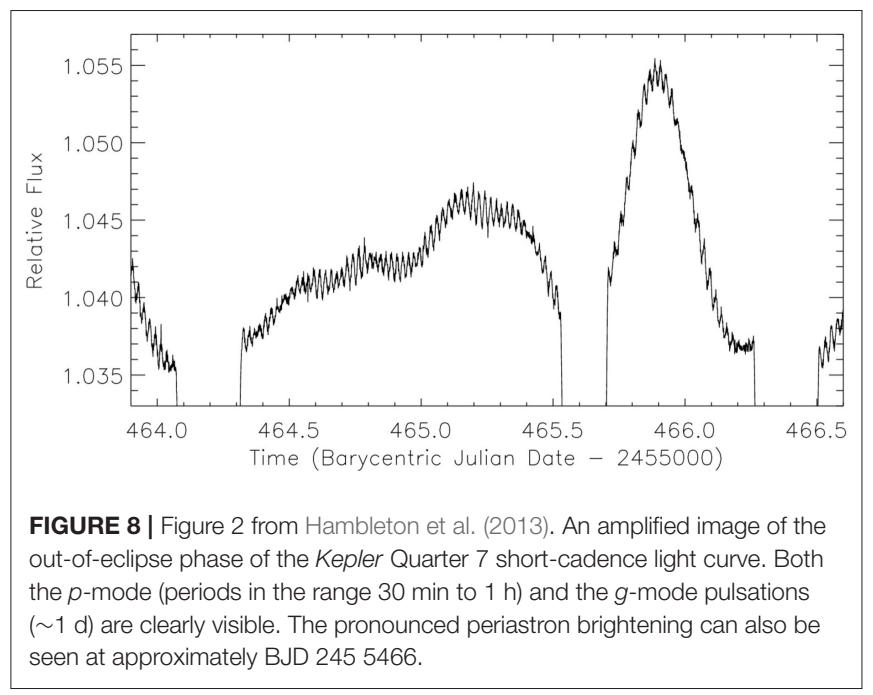

time over many years, these phenomena were not investigated comprehensively until the project of Bowman et al. (2016) using Kepler data from $983 \delta$ Sct stars observed continuously for 4 years. Bowman et al. found that $61.3 \%$ of stars in the sample showed amplitude variation in at least one pulsation mode during the 4 years. One star, KIC 7106205, showed a remarkable amplitude decrease for a single frequency over the first 2 years of the Kepler mission from 5 to $<1$ mmag (Bowman and Kurtz, 2014) (Figure 10). The amplitude of this same mode was found from WASP data to have decreased from 11 to $5 \mathrm{mmag}$ during the 2 years prior to the Kepler mission (Bowman et al., 2015). Bowman and Kurtz (2014) suggest that this dramatic decrease might be explained by non-linear mode coupling with energy transfer from the $p$ mode to low-frequency high-degree $g$ modes that are not visible because their light variations average out over the stellar disk.

\subsection{Finding and Interpreting Frequency (Period) Spacing Patterns-The Key to Asteroseismology}

Because $\delta$ Sct stars have modes of low radial order $n$, these modes cannot be treated using asymptotic pulsation theory and are not expected to show regular frequency spacings, such as the equal spacings between modes of consecutive radial order that are evident in, e.g., solar-like oscillators. This lack of obvious regular frequency patterns, plus the more rapid rotation in these stars compared to the Sun, leading to asymmetric splitting of the modes into overlapping multiplets, has made it nearly impossible to identify the pulsation modes with certainty. In addition, not all modes that are predicted by non-adiabatic pulsation calculations are seen in the amplitude spectrum. Nevertheless, Suárez et al. (2014), using a grid of stellar models and calculating average frequency separations for degree $\ell=1$ through 3 modes, show that an average large frequency spacing $(\Delta v)$ can be determined and used to derive the mean stellar density (Figure 11). García Hernández et al. (2015) subsequently 


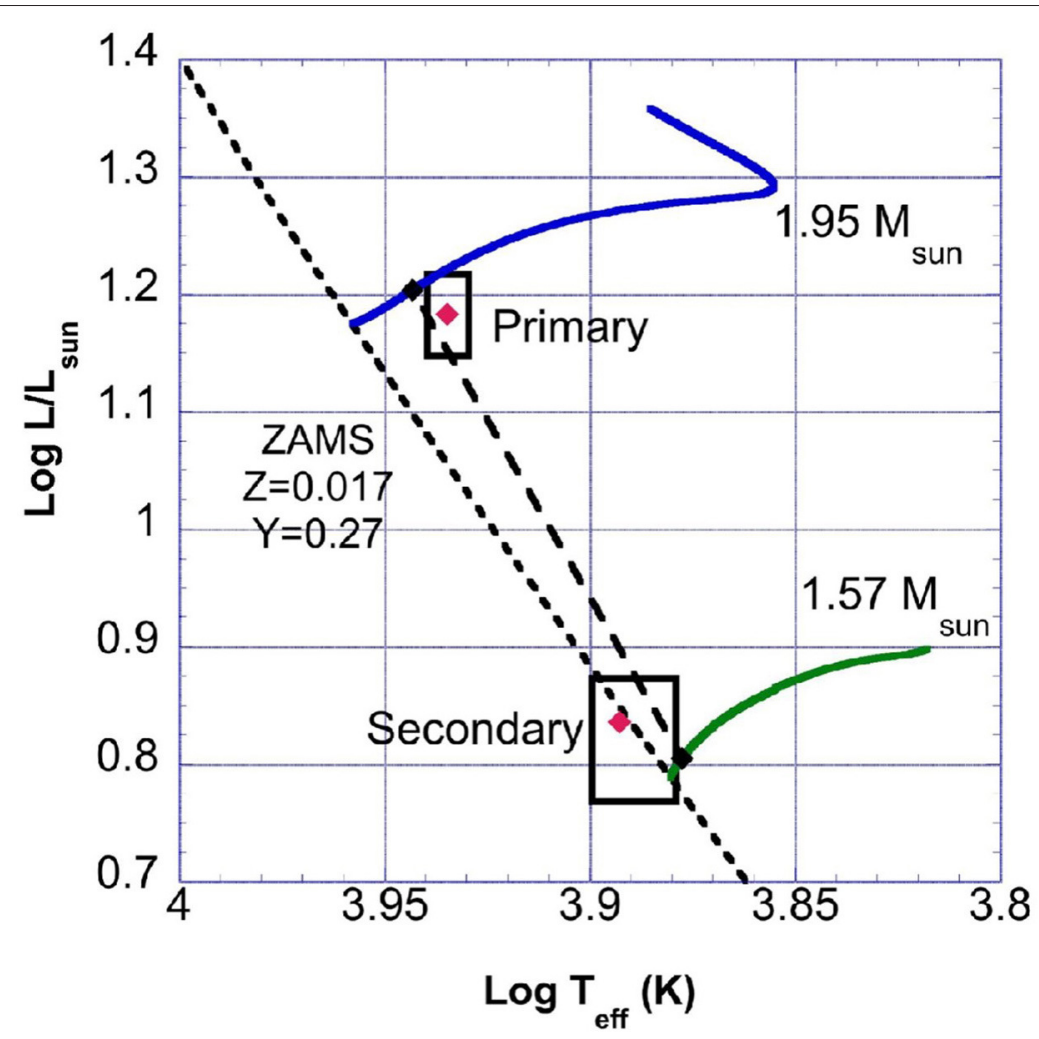

FIGURE 9 | Figure 13 from Hambleton et al. (2013). An H-R diagram for stellar models of components of KIC 4544587. The boxes outline the parameter space for the observationally derived primary and secondary components. The short-dashed line is zero-age main-sequence position for stellar models with $Z=0.017, \mathrm{Y}=$ 0.27. Also shown are evolutionary tracks for a 1.95- $\mathrm{M}_{\odot}$ (blue) and 1.57- $\mathrm{M}_{\odot}$ (green) model. The two models with the same age and composition closest to the observational constraints are connected by the long-dashed line. The red diamonds mark the best-fitting models for each star that do not have exactly the same age and composition.

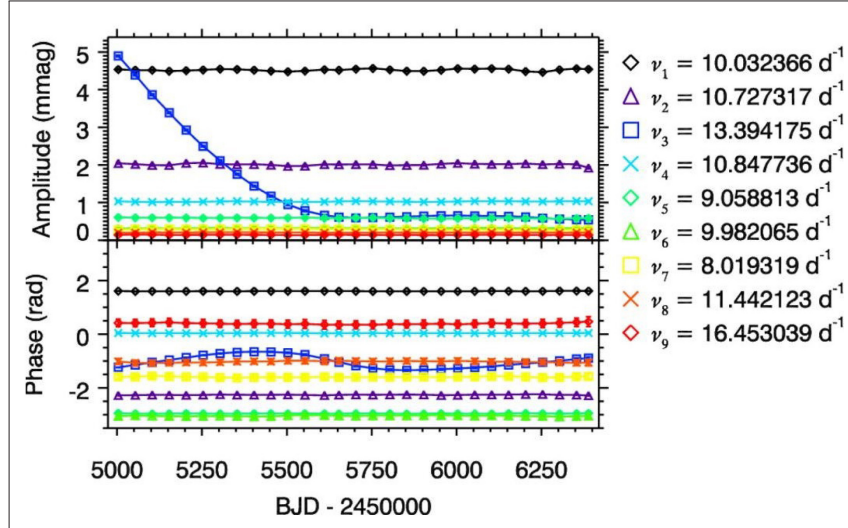

FIGURE 10 | Figure 8 from Bowman et al. (2016). KIC 7106205 amplitude and phase tracking plot showing variability in pulsation mode amplitudes and phases over time.

calibrated an observational frequency spacing - mean density relationship using eclipsing binaries with a $\delta$ Sct component observed by CoRoT and Kepler to determine independently the mean density (Figure 12).

Paparó et al. (2016a,b) noticed by eye, and then confirmed by algorithm, that one or more sequences of characteristic spacings

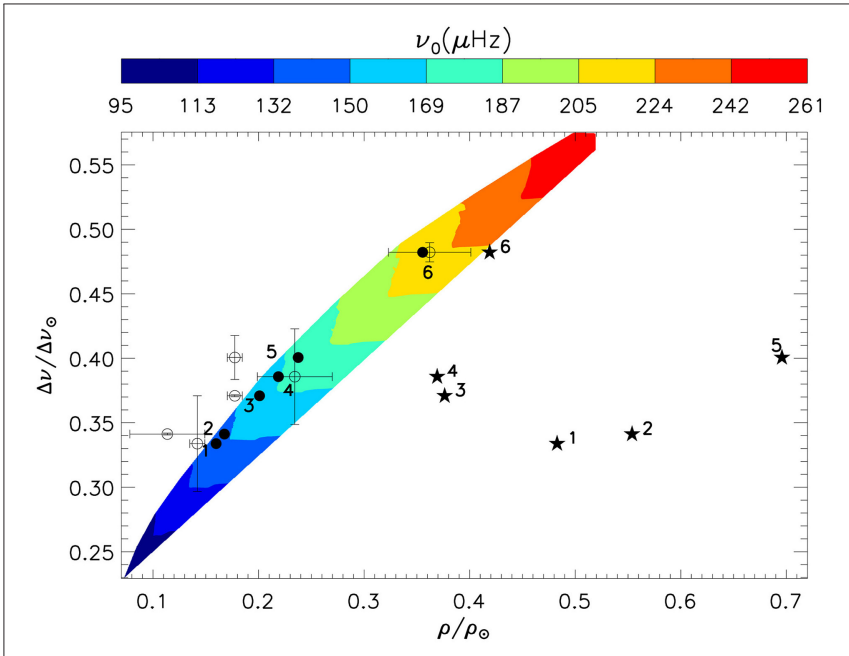

FIGURE 11 | Figure 2 from Suárez et al. (2014). Predicted large separation as a function of the mean density of the star, normalized to their solar values $134.8 \mu \mathrm{Hz}$ and $1.48 \mathrm{~g} \mathrm{~cm}^{-3}$, respectively. Color contours indicate the predicted frequency of the fundamental radial mode. Filled dots, empty dots, and star symbols represent mean densities found in Suárez et al. (2014), in the literature, and using the calibration of Tingley et al. (2011), respectively. For the sake of clarity, the error bars in star symbol estimates are omitted, since they are larger than the abscissa range. Reproduced with permission (C) ESO. 
could be found in a sample of $90 \delta$ Sct stars observed by CoRoT (Figures 13, 14). It is not always easy to determine, however, whether these characteristic frequency spacings are between successive radial order modes of the same angular degree (i.e., represent $\Delta v$ ) or are instead a combination of $\Delta v$ and the rotational splitting frequency.

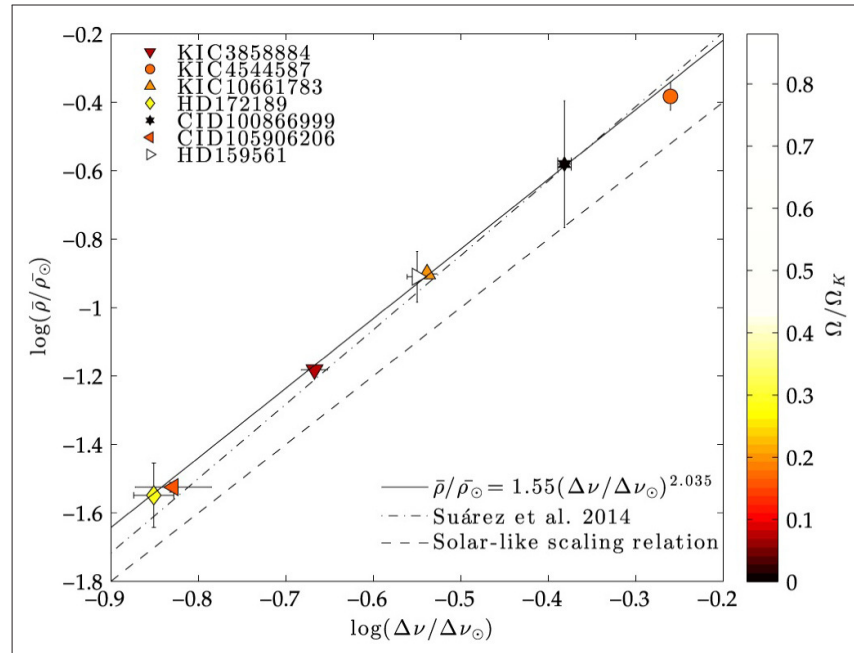

FIGURE 12 | Figure 1 from García Hernández et al. (2015). Large separation - mean density relation obtained for seven binary systems. A linear fit to the points is also depicted, as well as the solar-like scaling relation from Tassoul (1980), and the theoretical scaling relation for non-rotating models of $\delta$ Sct stars from Suárez et al. (2014). Symbols are plotted with a gradient color scale to account for the different rotation rates. (C) AAS. Reproduced with permission.

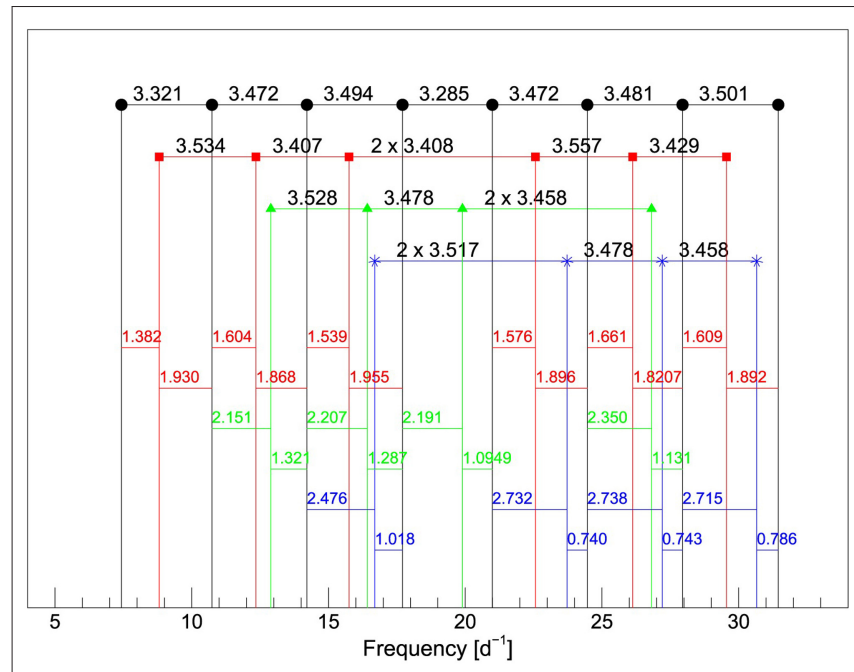

FIGURE 13 | Figure 1 from Paparó et al. (2016a). Sequences with quasi-equal spacing, and shifts of the sequences for CoRoT 102675756. First-black dots, average spacing $2.292 \pm 0.138 \mathrm{~d}^{-1}$; Second-red squares, $2.290 \pm 0.068$ $\mathrm{d}^{-1}$; Third-green triangles, $2.265 \pm 0.057 \mathrm{~d}^{-1}$; Fourth-blue stars, $2.242 \pm$ $0.051 d^{-1}$. The mean spacing of the star is $2.277 \pm 0.088 d^{-1}$. The shifts of the second, third, and fourth sequences relative to the first one are also given in the same color as the sequences. (C) AAS. Reproduced with permission.
While these methods allow one to use $\delta$ Sct frequencies to determine a characteristic spacing and mean density, and point toward mode identifications, i.e., being able to identify sequences of modes of the same angular degree, they fall short of finishing the goal of detailed mode identification for asteroseismology.

Fortunately, $\delta$ Sct stars early in their main-sequence lifetime have simpler patterns as their cores are less perturbed by changes in composition gradient at the convective core boundary that lead to "avoided crossings" and modes with mixed $p$ - and $g$-mode character. Bedding et al. (2020) found complete very regular sequences of $p$ modes among 60 young $\delta$ Sct variables observed by Kepler and TESS (see section 4) and was able to use these to identify the modes, with an assumption that the highest amplitude mode in about $1 / 3$ of the sample stars at frequency $18-23 \mathrm{~d}^{-1}$ likely is the radial fundamental $(n=1, \ell=0) p$ mode, finally opening a window for $\delta$ Sct asteroseismology. This task may have been made easier by many stars in the sample having relatively slow rotation or possibly being observed poleon, so that large rotational splittings did not confuse the sequence (Benkő and Paparó, 2020).

\subsection{0. $\gamma$ Doradus Breakthroughs}

A similar breakthrough for asteroseismology of $\gamma$ Doradus variables was enabled by the Kepler data. The $\gamma$ Dor $g$-mode periods are of high radial order, and should obey asymptotic period spacing relations. These modes are sensitive to conditions at the convective core boundary, where a composition gradient from hydrogen burning forms. This gradient is altered by mixing from convective overshooting and differential rotation. This composition gradient perturbs the expected even asymptotic period spacing, and causes mode trapping that shifts pulsation frequencies. These deviations from even period spacing can

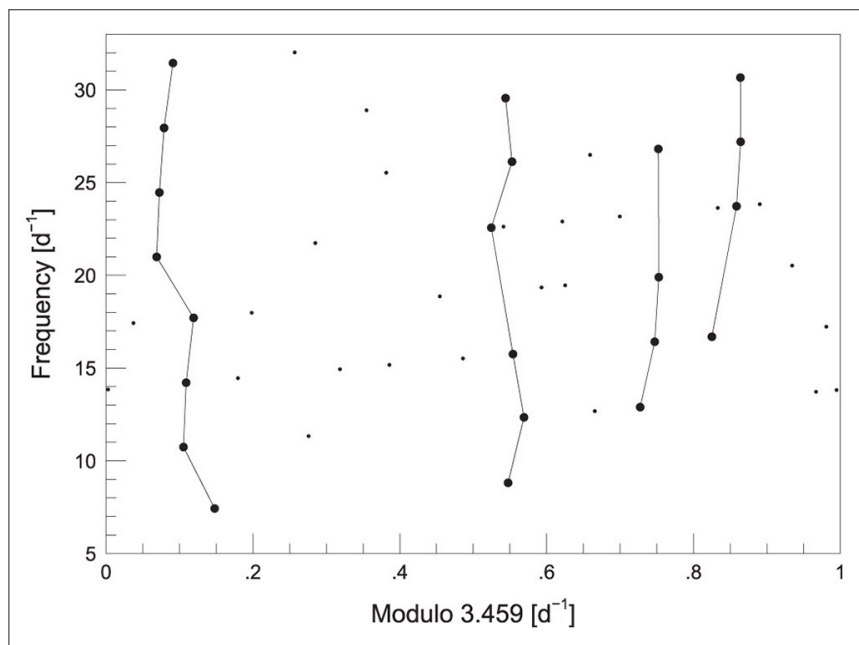

FIGURE 14 | Figure 2 from Paparó et al. (2016a). Echelle diagram for CoRoT 102675756, consistent with the sequences of the Figure $\mathbf{1 3}$ result from visual inspection. The mean spacing of the star was used as a modulo frequency. The whole frequency content of the star is plotted (small and large dots). The larger dots show the vertical representation of the sequences, the echelle ridges. (C) AAS. Reproduced with permission. 


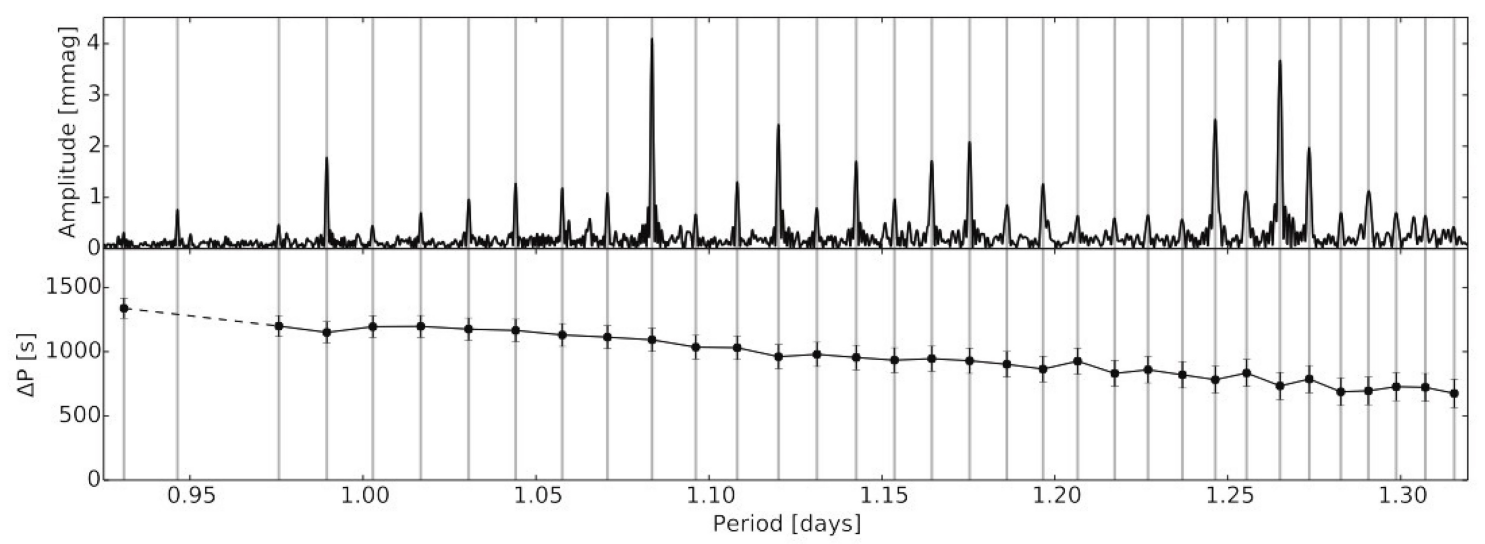

FIGURE 15 | Figure 11 from Van Reeth et al. (2015a). (Top) Close-up of part of the Fourier spectrum of KIC 11721304 (black). All the marked frequencies are accepted following the criterion described in Van Reeth et al. (2015a). (Bottom) The period spacing as computed from the accepted frequencies. The black markers and gray lines indicate the frequencies for which a smooth spacing pattern was found. Reproduced with permission @ ESO.

be used to probe the region of the convective-core boundary, constrain mixing profiles, and measure interior rotation rates (See theoretical and modeling papers predicting and explaining these effects by Miglio et al., 2008 and Bouabid et al., 2013).

Despite the expectation that rapid rotation and overlapping non-equidistant rotationally split multiplets would make it difficult to find a period-spacing pattern, Van Reeth et al. $(2015 a, b)$ were able to develop a period-spacing detection algorithm and successfully identify period spacings in several $\gamma$ Dor stars (Figure 15). As is the situation for the $\delta$ Sct frequency spacings, this method by itself does not allow one to identify the radial order $n$, angular degree $\ell$ or azimuthal order $m$ of the modes. Nevertheless, these techniques have been subsequently developed and applied for studies of $\gamma$ Dor stars observed by Kepler and CoRoT (see, e.g., Ouazzani et al., 2017, 2020; Li et al., 2019a,b).

In the $\gamma$ Dor literature, the gravity or $g$ modes are also referred to as gravito-inertial modes, as they are subject to both buoyancy and Coriolis forces because of rotation in the star (see, e.g., Mombarg et al., 2019). A key recent development for $\gamma$ Dor stars was the detection and exploitation of the $r$ modes, or global Rossby waves, (see, e.g., Saio et al., 2018) in many $\gamma$ Dor stars observed by Kepler. These modes consist of predominantly toroidal motions that do not have a restoring force or cause light variation in a non-rotating star because they cause no compression or expansion. However, in a rotating star, the toroidal motion couples with spheroidal motion caused by the Coriolis force, resulting in temperature perturbations that are visible. In $\gamma$ Dor stars, the $r$ modes of a given azimuthal order $m$ appear in groups with slightly lower frequency than $m$ times the rotation frequency. These modes can be excited by the $\kappa$ mechanism.

Gravity mode and inertial ( $r$ ) mode period spacing patterns found in Kepler $\gamma$ Dor stars, in some cases supplemented by $\delta$ Sct $p$ modes for the hybrid pulsators, have since been used by many groups to determine core and envelope rotation profiles in these stars (see, e.g., Van Reeth et al., 2016, 2018; Ouazzani et al., 2017;
Li et al., 2019b, 2020). Interior differential rotation has also been studied and confirmed in $\gamma$ Dor stars using the mode spacing patterns by, e.g., Aerts et al. (2017), Christophe et al. (2018), and Van Reeth et al. (2018).

Many important modeling advances for $\gamma$ Dor stars have resulted from the Kepler observations, among these models of individual targets (e.g., Kurtz et al., 2014; Saio et al., 2015; Schmid and Aerts, 2016). Mombarg et al. (2019, 2020) have developed comprehensive model grids to interpret mode spacing patterns for ensembles of $\gamma$ Dor stars and evaluate the effects of including diffusive settling and radiative levitation.

\subsection{1. $\delta$ Sct Discoveries in Binaries and Clusters}

While not discussed in detail here, the Kepler mission discovered many eclipsing binary systems. As mentioned earlier, the stellar properties derived independently from the binary system modeling can be used to calibrate asteroseismic techniques and supplement constraints for detailed asteroseismic models. A catalog of such systems discovered or observed by Kepler and K2 is maintained by Villanova University, with links to the Mikulski Archive for Space Telescopes (MAST), and the VizieR On-line Data Catalog (see, e.g., Kirk et al., 2016a,b). As of this writing, the Third Edition of this catalog lists 2,922 binaries observed during the original Kepler mission ${ }^{2}$ and 664 binaries from $\mathrm{K}^{3}$.

Many of these binaries have potential $\delta$ Sct (or hybrid) components. For example, Liakos and Niarchos (2016, 2017) catalog properties of 199 systems, many from Kepler observations, and Gaulme and Guzik (2019) find 149 such systems in the Kepler data. See also Kahraman Aliçavuş et al. (2017) who discuss spectroscopic observations of 92 eclipsing binaries with a $\delta$ Sct component apart from the Kepler data. We can add to this list 341 Kepler non-eclipsing A/F star binaries discovered from pulsation phase modulation by Murphy et al.

\footnotetext{
${ }^{2}$ http://keplerebs.villanova.edu

${ }^{3}$ http://keplerebs.villanova.edu/k2
} 
(2018a,b), and the 176 Kepler heartbeat stars mentioned earlier (Kirk et al., 2016b). There are a few dozen Kepler binary systems containing a $\delta$ Sct component that have been studied in detail to date. A search on the ADS abstract service with terms "binary" and " $\delta$ Sct" in the abstract, and "KIC" (Kepler Input Catalog) in the title gives 42 results, and shows a trend of increasing number of publications from two to ten per year over the past decade. Searching for publications on binary Kepler objects with " $\gamma$ Dor" in the abstract yields 28 results, with many of the objects studied being hybrid pulsators. This search does not include papers that are likely to appear soon studying binaries observed during the K2 mission.

$\delta$ Sct stars found in clusters have similar advantages to those in binaries, as the common age and metallicity of the cluster members offer independent constraints. For example, a comprehensive paper by Sandquist et al. (2016) compares the age and distance modulus of the open cluster NGC 6811 derived using a variety of methods. This cluster was observed during the original Kepler mission, and found to contain an eclipsing binary with an Am star and $\gamma$ Dor component, many pulsating stars near the cluster turnoff, including $28 \delta$ Sct, $15 \gamma$ Dor, and 5 hybrid stars, and many giant stars, some on the asymptotic giant branch and some in the "red clump" core helium-burning phase. They model the binary to determine component masses and compare with isochrones, finding inconsistent ages between the components of $1.05 \mathrm{Gyr}$ for the Am star primary, and 1.21 Gyr for the $\gamma$ Dor secondary; the younger Am star age is more consistent with the 1.0 Gyr age derived from the main-sequence turnoff in the color-magnitude diagram. However, the Am star abundance peculiarities are not properly taken into account in stellar models, so the Am star age may be more suspect. In addition, the (near-solar) metallicity of the stars in the cluster is uncertain and the ages would be less discrepant with a slightly lower metallicity than adopted. Applying asteroseismic $\Delta v$ vs. $v_{\max }$ relations for the core helium-burning stars to determine their radii and masses, they find that the derived masses of these stars appear to be larger (or their radii smaller) than expected for the cluster age(s) derived from the binary. These stars are more consistent with a 0.9 Gyr age. They derive the distance modulus using the period-luminosity relationship of high-amplitude $\delta$ Sct stars, finding $(m-M)_{V}=10.37 \pm$ 0.03 , which is lower than the value derived using the eclipsing binary, $(m-M)_{V}=10.47 \pm 0.05$. This example shows the possibilities for combining multiple constraints from clusters to check for consistency in inferences, and to identify discrepancies in modeling or asteroseismic analysis techniques for a particular star class.

\section{SUCCESSES, UNRESOLVED PROBLEMS, AND QUESTIONS}

In summary, it would not be an understatement to conclude that the Kepler mission has revolutionized the field of asteroseismology, in particular for $\delta$ Sct stars, and given researchers a wealth of data for analyses, modeling efforts, and motivating future long-term observations. A non-exhaustive list of successes includes:
- Unprecedented long time-series (months to years), highcadence (1 or $30 \mathrm{~min}$ ), high-precision photometry for thousands of $\delta$ Sct, $\gamma$ Dor, and hybrid variables, many newly discovered using the Kepler data.

- Interpretation and application of frequency and period spacings and patterns to inform mode identification, exploiting these to determine interior structure, extent of mixing, and rotation profiles.

- More definitive quantification of the pervasiveness and range of amplitude variations that await explanation.

- Large expansion in the number of binaries showing tidally excited modes, establishing a new class of variable stars.

- Motivation for exploration of additional pulsation driving mechanisms, advancing stellar pulsation theory.

- More definitive data to quantify the role of magnetic fields in pulsation.

- More definitive constraints for quantifying the effects of element diffusive settling and radiative levitation and accurately including these processes in stellar models.

There are many problems and questions motivated or amplified by the Kepler data, among these:

- Why are many of the pulsation modes predicted by linear pulsation theory not observed?

- What is the origin of the low frequencies found in many $\delta$ Sct stars?

- Can observed frequency spacings and patterns be interpreted and used for mode identification?

- What determines the amplitudes of $\delta$ Sct modes, and what causes amplitude variations?

- Why are some stars in the $\delta$ Sct (and $\gamma$ Dor) instability regions "constant," i.e., not pulsating?

- Why are some chemically peculiar stars pulsating?

- Are HADS or SX Phe stars different from each other or from normal $\delta$ Sct stars?

- What is the origin of blue stragglers?

- What is the origin of magnetic activity, spots, and flares in hot stars?

- What is the origin of the abundance peculiarities in $\lambda$ Boo, Am, and Ap stars?

- Can new proposed pulsation driving mechanisms explain the unexpected frequencies observed in some $\delta$ Sct stars?

Answering these questions will require long-term monitoring, directed campaigns, high-resolution spectroscopy, multicolor photometry, interferometry, and other observations, in addition to advances in stellar evolution and pulsation theory and modeling.

\section{THE NEAR FUTURE AND TESS}

While the Kepler spacecraft ended its K2 mission in November 2018, the NASA TESS spacecraft (Ricker et al., 2015) was launched in April 2018. The TESS spacecraft has some advantages and some disadvantages compared to Kepler for asteroseismology. The TESS mission is surveying more of the sky over its mission lifetime, while Kepler covered a single field of view in the Cygnus and Lyra constellations during its 
original mission, and 18 fields along the ecliptic during the extended K2 mission. However, TESS observes a sector of the sky continuously for only 27 days, compared to the possibility of obtaining up to 4 years of nearly continuous data during the original Kepler mission, or nearly 3 months continuously during K2. TESS is collecting full-frame images every 30 min (every 10 min starting in Cycle 3), and also has the possibility for 2 min and even 20-s cadence observations for selected targets. The pixel size for the TESS cameras is larger, making crowding and contamination from nearby stars in the field an issue that must be taken into account in data analyses. The redder TESS bandpass reduces the observed amplitudes of $\delta$ Sct pulsations by about $25 \%$ compared to the amplitudes of the Kepler mission (Antoci et al., 2019).

The TESS first-light papers have been published, including a first view of $\delta$ Sct and $\gamma$ Dor stars with the TESS mission (Antoci et al., 2019). This paper contains up-to-date descriptions of $\delta$ Sct and related stars, including pre-main-sequence $\delta$ Sct stars that were not studied in detail using Kepler observations, $\lambda$ Boo stars not discussed in this review, TESS observations of very bright stars such as $\alpha$ Pic, and the pulsation class prototypes SX Phe (see also Daszyńska-Daszkiewicz et al., 2020a,b) and $\gamma$ Dor that were not targeted by Kepler. The paper also has an extensive explanation of the role of turbulent pressure in the hydrogen ionization zone in driving $\delta$ Sct pulsations, especially in the context of Am stars that are expected to have helium depleted from diffusive settling, inhibiting the classical $\kappa$-effect pulsation driving mechanism. TESS observations will extend and enhance the Kepler legacy.

What can we expect for the future of Kepler and TESS observations? Asteroseismic analyses will be conducted using data from individual stars or ensembles of stars with common properties, binaries, and $\delta$ Sct stars in clusters. K2 has observed many open clusters on the ecliptic that contain $\delta$ Sct stars (e.g., Hyades, Praesepe, Pleiades, and M67). Studies of clusters show promise to finally understand the nature of blue stragglers, and the development of abundance peculiarities in Am and $\lambda$ Boo stars. There were no pre-main-sequence $\delta$ Sct stars in the original Kepler field, but discoveries for these stars may await using K2 or TESS data. It is hoped that these data will help disentangle or systematize the picture for stellar interior

\section{REFERENCES}

Aerts, C., Christensen-Dalsgaard, J., and Kurtz, D. W. (2010). Asteroseismology. Dordrecht: Springer Astronomy and Astrophysics Library. doi: 10.1007/978-1-4020-5803-5

Aerts, C., Van Reeth, T., and Tkachenko, A. (2017). The interior angular momentum of core hydrogen burning stars from gravity-mode oscillations. Astrophys. J. Lett. 847:L7. doi: 10.3847/2041-8213/aa8a62

Antoci, V., Cunha, M., Houdek, G., Kjeldsen, H., Trampedach, R., Handler, G., et al. (2014). The role of turbulent pressure as a coherent pulsational driving mechanism: the case of the $\delta$ Scuti Star HD 187547. Astrophys. J. 796:118. doi: 10.1088/0004-637X/796/2/118

Antoci, V., Cunha, M. S., Bowman, D. M., Murphy, S. J., Kurtz, D. W., Bedding, T. R., et al. (2019). The first view of $\delta$ Scuti and $\gamma$ Doradus stars with the TESS mission. Mon. Not. RAS 490, 4040-4059. doi: 10.1093/mnras/s tz2787 and evolution modeling from the pre-main-sequence through the shell H-burning stage, for example, the roles of processes such as convective overshooting, differential rotation, angular momentum transport, element levitation and settling, magnetic fields, mixing from internal gravity waves, etc. It is hoped that advances in theory and multidimensional stellar modeling, e.g., non-radial, non-linear, non-adiabatic pulsation modeling including turbulent and magnetic pressure and energy or rapid differential rotation, will lead to explanations for pulsation mode driving, mode selection, and amplitudes, and will better define instability strip boundaries.

\section{AUTHOR CONTRIBUTIONS}

JG was the sole author of this article that reviews the contributions of many authors to the field of $\delta$ Sct asteroseismology using Kepler data.

\section{FUNDING}

JG's research was supported at Los Alamos National Laboratory (LANL), managed by Triad National Security, LLC for the U.S. Department of Energy's NNSA, Contract \#89233218CNA000001. JG also gratefully acknowledges a Los Alamos National Laboratory Center for Space and Earth Sciences Rapid Response grant for Summer 2020.

\section{ACKNOWLEDGMENTS}

JG thanks editors K. Kinemuchi and A. Baran for the opportunity to write this review, and for their careful reading and suggestions. JG also thanks many colleagues who have made important contributions to this field. In addition, JG thanks the two reviewers, and also Simon Murphy, who have provided extensive suggestions for additional literature to take into account, and for sharing their perspectives on the developments in this field. JG thanks colleagues J. Jackiewicz and P. Bradley for advice and help with the Kepler data over many years. Finally, JG thanks the authors and journals for granting permission to reproduce the figures used in this paper.

Antoci, V., Handler, G., Campante, T. L., Thygesen, A. O., Moya, A., Kallinger, T., et al. (2011). The excitation of solar-like oscillations in a $\delta$ Sct star by efficient envelope convection. Nature 477, 570-573. doi: 10.1038/nature 10389

Balona, L. A. (2012). Kepler observations of flaring in A-F type stars. Mon. Not. RAS 423, 3420-3429. doi: 10.1111/j.1365-2966.2012.21135.x

Balona, L. A. (2013). Activity in A-type stars. Mon. Not. RAS 431, 2240-2252. doi: $10.1093 / \mathrm{mnras} / \mathrm{stt} 322$

Balona, L. A. (2014). Low frequencies in Kepler $\delta$ Scuti stars. Mon. Not. RAS 437, 1476-1484. doi: 10.1093/mnras/stt1981

Balona, L. A. (2015). Flare stars across the H-R diagram. Mon. Not. RAS 447, 2714-2725. doi: 10.1093/mnras/stu2651

Balona, L. A. (2017). Starspots on A stars. Mon. Not. RAS 467, 1830-1837. doi: $10.1093 / \mathrm{mnras} / \mathrm{stx} 265$

Balona, L. A. (2018). Pulsation in intermediate-mass stars. Front. Astron. Space Sci. 5:43. doi: $10.3389 /$ fspas. 2018.00043 
Balona, L. A. (2019). Evidence for spots on hot stars suggests major revision of stellar physics. Mon. Not. RAS 490, 2112-2116. doi: 10.1093/mnras/stz2808

Balona, L. A., Catanzaro, G., Abedigamba, O. P., Ripepi, V., and Smalley, B. (2015a). Spots on Am stars. Mon. Not. RAS 448, 1378-1388. doi: $10.1093 / \mathrm{mnras} / \mathrm{stv} 076$

Balona, L. A., Daszyńska-Daszkiewicz, J., and Pamyatnykh, A. A. (2015b). Pulsation frequency distribution in $\delta$ Scuti stars. Mon. Not. RAS 452, 3073-3084. doi: 10.1093/mnras/stv1513

Balona, L. A., Guzik, J. A., Uytterhoeven, K., Smith, J. C., Tenenbaum, P., and Twicken, J. D. (2011a). The Kepler view of $\gamma$ Doradus stars. Mon. Not. RAS 415, 3531-3538. doi: 10.1111/j.1365-2966.2011.18973.x

Balona, L. A., and Nemec, J. M. (2012). A search for SX Phe stars among Kepler $\delta$ Scuti stars. Mon. Not. RAS 426, 2413-2418. doi: 10.1111/j.1365-2966.2012.21957.x

Balona, L. A., Ripepi, V., Catanzaro, G., Kurtz, D. W., Smalley, B., De Cat, P., et al. (2011b). Kepler observations of Am stars. Mon. Not. RAS 414, 792-800. doi: 10.1111/j.1365-2966.2011.18454.x

Bedding, T. R., Murphy, S. J., Hey, D. R., Huber, D., Li, T., Smalley, B., et al. (2020). Very regular high-frequency pulsation modes in young intermediate-mass stars. Nature 581, 147-151. doi: 10.1038/s41586-020-2226-8

Benkö, J. M., and Paparó, M. (2020). A glimpse inside $\delta$ Scuti stars. Nature 581, 141-142. doi: 10.1038/d41586-020-01169-z

Borucki, W. J., Koch, D., Basri, G., Batalha, N., Brown, T., Caldwell, D., et al. (2010). Kepler planet-detection mission: introduction and first results. Science 327:977. doi: 10.1126/science.1185402

Bouabid, M. P., Dupret, M. A., Salmon, S., Montalbán, J., Miglio, A., and Noels, A. (2013). Effects of the Coriolis force on high-order g modes in $\gamma$ Doradus stars. Mon. Not. RAS 429, 2500-2514. doi: 10.1093/mnras/sts517

Bowman, D. M. (2017). Amplitude Modulation of Pulsation Modes in Delta Scuti Stars. Springer Theses. Cham: Springer International Publishing AG doi: 10.1007/978-3-319-66649-5

Bowman, D. M., Buysschaert, B., Neiner, C., Pápics, P. I., Oksala, M. E., and Aerts, C. (2018). K2 space photometry reveals rotational modulation and stellar pulsations in chemically peculiar A and B stars. Astron. Astrophys. 616:A77. doi: 10.1051/0004-6361/201833037

Bowman, D. M., Holdsworth, D. L., and Kurtz, D. W. (2015). Combining WASP and Kepler data: the case of the $\delta$ Sct star KIC 7106205. Mon. Not. RAS 449, 1004-1010. doi: 10.1093/mnras/stv364

Bowman, D. M., and Kurtz, D. W. (2014). Pulsational frequency and amplitude modulation in the $\delta$ Sct star KIC 7106205. Mon. Not. RAS 444, 1909-1918. doi: $10.1093 / \mathrm{mnras} / \mathrm{stu} 1583$

Bowman, D. M., and Kurtz, D. W. (2018). Characterizing the observational properties of $\delta$ Sct stars in the era of space photometry from the Kepler mission. Mon. Not. RAS 476:3169-3184. doi: 10.1093/mnras/sty449

Bowman, D. M., Kurtz, D. W., Breger, M., Murphy, S. J., and Holdsworth, D. L. (2016). Amplitude modulation in $\delta$ Sct stars: statistics from an ensemble study of Kepler targets. Mon. Not. RAS 460, 1970-1989. doi: 10.1093/mnras/stw1153

Bradley, P. A., Guzik, J. A., Miles, L. F., Uytterhoeven, K., Jackiewicz, J., and Kinemuchi, K. (2015). Results of a search for $\gamma$ Dor and $\delta$ Sct stars with the Kepler spacecraft. Astron. J. 149:68. doi: 10.1088/0004-6256/149/2/68

Bradley, P. A., Guzik, J. A., Miles, L. F., Uytterhoeven, K., Jackiewicz, J., and Kinemuchi, K. (2016). Erratum: "Results of a search for $\gamma$ Dor and $\delta$ Sct stars with the Kepler spacecraft" (2015, AJ 149:68). Astron. J. 151:86. doi: 10.3847/0004-6256/151/3/86

Breger, M. (1980). Delta-Scuti stars and Swarf Cepheids-review and pulsation modes. Space Sci. Rev. 27, 361-370. doi: 10.1017/S025292110008177X

Breger, M., Lenz, P., Antoci, V., Guggenberger, E., Shobbrook, R. R., Handler, G., et al. (2005). Detection of $75+$ pulsation frequencies in the $\delta$ Scuti star FG Virginis. Astron. Astrophys. 435, 955-965. doi: 10.1051/0004-6361:20 042480

Breger, M., and Montgomery, M. (Eds.). (2000). Delta Scuti and Related Stars, Volume 210 of Astronomical Society of the Pacific Conference Series. San Francisco, CA: Astronomical Society of the Pacific.

Breger, M., Montgomery, M. H., Lenz, P., and Pamyatnykh, A. A. (2017). Nonradial and radial period changes of the $\delta$ Scuti star $4 \mathrm{CVn}$. II. Systematic behavior over 40 years. Astron. Astrophys. 599:A116. doi: $10.1051 / 0004-6361 / 201629797$
Brun, A. S., Browning, M. K., and Toomre, J. (2005). Simulations of core convection in rotating A-type stars: magnetic dynamo action. Astrophys. J. 629, 461-481. doi: 10.1086/430430

Buysschaert, B., Neiner, C., Martin, A. J., Aerts, C., Bowman, D. M., Oksala, M. E., et al. (2018). Detection of magnetic fields in chemically peculiar stars observed with the K2 space mission. Mon. Not. RAS 478, 2777-2793. doi: $10.1093 / \mathrm{mnras} / \mathrm{sty} 1190$

Cheng, S. J., Fuller, J., Guo, Z., Lehman, H., and Hambleton, K. (2020). Detailed characterization of heartbeat stars and their tidally excited oscillations. Astrophys. J. 903:122. doi: 10.3847/1538-4357/abb46d

Christophe, S., Ballot, J., Ouazzani, R. M., Antoci, V., and Salmon, S. J. A. J. (2018). Deciphering the oscillation spectrum of $\gamma$ Doradus and SPB stars. Astron. Astrophys. 618:A47. doi: 10.1051/0004-6361/201832782

Cox, A. N. (1983). "Stability problems with an application to early-type stars," in Saas-Fee Advanced Course 13: Astrophysical Processes in Upper Main Sequence Stars, eds A. N. Cox, S. Vauclair, and J. P. Zahn (Geneva: Geneva Observatory),

Daszyńska-Daszkiewicz, J., Pamyatnykh, A. A., Walczak, P., and Szewczuk, W. (2020a). Seismic analysis of the double-mode radial pulsator SX Phoenicis. Mon. Not. RAS 499, 3034-3045. doi: 10.1093/mnras/staa3056

Daszyńska-Daszkiewicz, J., Walczak, P., and Pamyatnykh, A. (2017). "On possible explanations of pulsations in Maia stars," in European Physical Journal Web of Conferences, Volume 160 of European Physical Journal Web of Conferences (Vienna), 03013. doi: 10.1051/epjconf/201716003013

Daszyńska-Daszkiewicz, J., Walczak, P., Pamyatnykh, A., Szewczuk, W., and Dziembowski, W. (2020b). "The complex asteroseismology of SX Phoenicis," in Stars and Their Variability Observed From Space, eds C. Neiner, W. W. Weiss, D. Baade, R. E. Griffin, C. C. Lovekin, and A. F. J. Moffat (San Francisco, CA: Astronomical Society of the Pacific), 81-85.

Dupret, M. A., Grigahcène, A., Garrido, R., Gabriel, M., and Scuflaire, R. (2005). Convection-pulsation coupling. II. Excitation and stabilization mechanisms in $\delta$ Sct and $\gamma$ Dor stars. Astron. Astrophys. 435, 927-939. doi: 10.1051/0004-6361:20041817

Featherstone, N. A., Browning, M. K., Brun, A. S., and Toomre, J. (2009). Effects of fossil magnetic fields on convective core dynamos in A-type stars. Astrophys. J. 705, 1000-1018. doi: 10.1088/0004-637X/705/1/1000

Fuller, J. (2017). Heartbeat stars, tidally excited oscillations and resonance locking. Mon. Not. RAS 472, 1538-1564. doi: 10.1093/mnras/stx2135

Gaia Collaboration, Brown, A. G. A., Vallenari, A., Prusti, T., de Bruijne, J. H. J., Babusiaux, C., et al. (2018). Gaia Data Release 2. Summary of the contents and survey properties. Astron. Astrophys. 616:A1. doi: 10.1051/0004-6361/201833051

García Hernández, A., Martín-Ruiz, S., Monteiro, M. J. P. F. G., Suárez, J. C., Reese, D. R., Pascual-Granado, J., et al. (2015). Observational $\delta v-\rho$ relation for $\delta$ Sct stars using eclipsing binaries and space photometry. Astrophys. J. Lett. 811:L29. doi: 10.1088/2041-8205/811/2/L29

García, R. A., and Ballot, J. (2019). Asteroseismology of solar-type stars. Liv. Rev. Sol. Phys. 16:4. doi: 10.1007/s41116-019-0020-1

Gaulme, P., and Guzik, J. A. (2019). Systematic search for stellar pulsators in the eclipsing binaries observed by Kepler. Astron. Astrophys. 630:A106. doi: 10.1051/0004-6361/201935821

Gilliland, R. L., Brown, T. M., Christensen-Dalsgaard, J., Kjeldsen, H., Aerts, C., Appourchaux, T., et al. (2010). Kepler asteroseismology program: introduction and first results. Publ. ASP 122:131. doi: 10.1086/650399

Grigahcène, A., Antoci, V., Balona, L., Catanzaro, G., Daszyńska-Daszkiewicz, J., Guzik, J. A., et al. (2010). Hybrid $\gamma$ Doradus- $\delta$ Scuti pulsators: new insights into the physics of the oscillations from Kepler observations. Astrophys. J. Lett. 713, L192-L197. doi: 10.1088/2041-8205/713/2/L192

Guo, Z., Shporer, A., Hambleton, K., and Isaacson, H. (2020). Tidally excited oscillations in heartbeat binary stars: pulsation phases and mode identification. Astrophys. J. 888:95. doi: 10.3847/1538-4357/ab58c2

Guzik, J. A., Bradley, P. A., Jackiewicz, J., Molenda-Zakowicz, J., Uytterhoeven, K., and Kinemuchi, K. (2015a). The occurrence of non-pulsating stars in the $\gamma$ Dor and $\delta$ Sct pulsation instability regions: results from Kepler quarter 14-17 data. Astron. Rev. 11, 1-24. doi: 10.1080/21672857.2015.1023120

Guzik, J. A., Bradley, P. A., Jackiewicz, J., Molenda-Zakowicz, J., Uytterhoeven, K., and Kinemuchi, K. (2015b). The occurrence of non-pulsating stars in the 
gamma Dor and delta Sct pulsation instability regions: results from Kepler quarter 14-17 data. arXiv 1502.00175.

Guzik, J. A., Bradley, P. A., Jackiewiecz, J., Uytterhoeven, K., and Kinemuchi, K. (2013). The occurrence of non-pulsating stars in the gamma Doradus/delta Scuti pulsation instability region. Astron. Rev. 8, 83-107. doi: 10.1080/21672857.2013.11519724

Guzik, J. A., Bradley, P. A., Jackiewiecz, J., Uytterhoeven, K., and Kinemuchi, K. (2014). The occurrence of non-pulsating stars in the gamma Doradus/delta Scuti pulsation instability region. Astron. Rev. 9, 41-65. doi: 10.1080/21672857.2014.11519730

Guzik, J. A., Garcia, J. A., and Jackiewicz, J. (2019). Properties of 249 delta Scuti variable star candidates observed during the NASA K2 mission. Front. Astron. Space Sci. 6:40. doi: 10.3389/fspas.2019.00040

Guzik, J. A., Jackiewicz, J., Pigulski, A., Catanzaro, G., Soukup, M. S., Gaulme, P., et al. (2020). Data analysis of bright main-sequence A- and B-type stars observed using the TESS and BRITE spacecraft. arXiv.

Guzik, J. A., Kaye, A. B., Bradley, P. A., Cox, A. N., and Neuforge, C. (2000). Driving the gravity-mode pulsations in $\gamma$ Doradus variables. Astrophys. J. Lett. 542, L57-L60. doi: 10.1086/312908

Hambleton, K., Fuller, J., Thompson, S., Prša, A., Kurtz, D. W., Shporer, A., et al. (2018). KIC 8164262: a heartbeat star showing tidally induced pulsations with resonant locking. Mon. Not. RAS 473, 5165-5176. doi: 10.1093/mnras/stx2673

Hambleton, K. M., Kurtz, D. W., Prša, A., Guzik, J. A., Pavlovski, K., Bloemen, S., et al. (2013). KIC 4544587: an eccentric, short-period binary system with $\delta$ Sct pulsations and tidally excited modes. Mon. Not. RAS 434, 925-940. doi: $10.1093 / \mathrm{mnras} / \mathrm{stt} 886$

Handler, G., and Shobbrook, R. R. (2002). On the relationship between the $\delta$ Scuti and $\gamma$ Doradus pulsators. Mon. Not. RAS 333, 251-262. doi: 10.1046/j.1365-8711.2002.05401.x

Holdsworth, D. L. (2021). The roAp stars observed by the Kepler space telescope. Front. Astron. Space Sci. doi: 10.3389/fspas.2021.626398

Howell, S. B., Sobeck, C., Haas, M., Still, M., Barclay, T., Mullally, F., et al. (2014). The K2 mission: characterization and early results. Publ. ASP 126:398. doi: $10.1086 / 676406$

Kahraman Aliçavuş, F., Soydugan, E., Smalley, B., and Kubát, J. (2017). Eclipsing binary stars with a $\delta$ Scuti component. Mon. Not. RAS 470, 915-931. doi: 10.1093/mnras/stx1241

Kirk, B., Conroy, K., Prsa, A., Abdul-Masih, M., Kochoska, A., Matijevic, G., et al. (2016a). VizieR Online Data Catalog: Kepler Mission. VII. Eclipsing Binaries in DR3 (Kirk+ 2016). Strasbourg: VizieR Online Data Catalog.

Kirk, B., Conroy, K., Prša, A., Abdul-Masih, M., Kochoska, A., Matijevič, G., et al. (2016b). Kepler eclipsing binary stars. VII. The catalog of eclipsing binaries found in the entire Kepler data set. Astron. J. 151:68. doi: 10.3847/0004-6256/151/3/68

Koch, D. G., Borucki, W. J., Basri, G., Batalha, N. M., Brown, T. M., Caldwell, D., et al. (2010). Kepler mission design, realized photometric performance, and early science. Astrophys. J. Lett. 713, L79-L86. doi: 10.1088/2041-8205/713/2/L79

Kurtz, D. W. (1982). Rapidly oscillating AP stars. Mon. Not. RAS 200, 807-859. doi: 10.1093/mnras/200.3.807

Kurtz, D. W., Saio, H., Takata, M., Shibahashi, H., Murphy, S. J., and Sekii, T. (2014). Asteroseismic measurement of surface-to-core rotation in a main-sequence A star, KIC 11145123. Mon. Not. RAS 444, 102-116. doi: 10.1093/mnras/stu1329

Li, G., Bedding, T. R., Murphy, S. J., Van Reeth, T., Antoci, V., and Ouazzani, R.-M. (2019a). Period spacings of $\gamma$ Doradus pulsators in the Kepler field: detection methods and application to 22 slow rotators. Mon. Not. RAS 482, 1757-1785. doi: $10.1093 / \mathrm{mnras} / \mathrm{sty} 2743$

Li, G., Van Reeth, T., Bedding, T. R., Murphy, S. J., and Antoci, V. (2019b). Period spacings of $\gamma$ Doradus pulsators in the Kepler field: Rossby and gravity modes in 82 stars. Mon. Not. RAS 487, 782-800. doi: 10.1093/mnras/stz1171

Li, G., Van Reeth, T., Bedding, T. R., Murphy, S. J., Antoci, V., Ouazzani, R.-M., et al. (2020). Gravity-mode period spacings and near-core rotation rates of $611 \gamma$ Doradus stars with Kepler. Mon. Not. RAS 491, 3586-3605. doi: $10.1093 / \mathrm{mnras} / \mathrm{stz} 2906$

Liakos, A., and Niarchos, P. (2016). Poetry in motion: asteroseismology of delta Scuti stars in binaries using Kepler data. arXiv 1606.08638.
Liakos, A., and Niarchos, P. (2017). Catalogue and properties of $\delta$ Scuti stars in binaries. Mon. Not. RAS 465, 1181-1200. doi: 10.1093/mnras/stw2756

Mathys, G., Kurtz, D. W., and Holdsworth, D. L. (2020). Long-period Ap stars discovered with TESS data. Astron. Astrophys. 639:A31. doi: 10.1051/0004-6361/202038007

Matthews, J. M. (2007). One small satellite, so many light curves: Examples of $\delta$ Scuti asteroseismology from the MOST space mission. Commun. Asteroseismol. 150:333. doi: 10.1553/cia150s333

McNamara, D. H. (2000). "The high-amplitude $\delta$ Scuti stars," in Delta Scuti and Related Stars, Volume 210 of Astronomical Society of the Pacific Conference Series, eds M. Breger, and M. Montgomery (San Francisco, CA), 373.

Miglio, A., Montalbán, J., Noels, A., and Eggenberger, P. (2008). Probing the properties of convective cores through g modes: high-order G modes in SPB and $\gamma$ Doradus stars. Mon. Not. RAS 386, 1487-1502. doi: 10.1111/j.1365-2966.2008.13112.x

Mombarg, J. S. G., Dotter, A., Van Reeth, T., Tkachenko, A., Gebruers, S., and Aerts, C. (2020). Asteroseismic modeling of gravity modes in slowly rotating A/F stars with radiative levitation. Astrophys. J. 895:51. doi: 10.3847/1538-4357/ab8d36

Mombarg, J. S. G., Van Reeth, T., Pedersen, M. G., Molenberghs, G., Bowman, D. M., Johnston, C., et al. (2019). Asteroseismic masses, ages, and core properties of $\gamma$ Doradus stars using gravito-inertial dipole modes and spectroscopy. Mon. Not. RAS 485, 3248-3263. doi: 10.1093/mnras/stz501

Murphy, S. J., Bedding, T. R., Niemczura, E., Kurtz, D. W., and Smalley, B. (2015). A search for non-pulsating, chemically normal stars in the $\delta$ Scuti instability strip using Kepler data. Mon. Not. RAS 447, 3948-3959. doi: $10.1093 / \mathrm{mnras} / \mathrm{stu} 2749$

Murphy, S. J., Hey, D., Van Reeth, T., and Bedding, T. R. (2019). Gaia-derived luminosities of Kepler A/F stars and the pulsator fraction across the $\delta$ Scuti instability strip. Mon. Not. RAS 485, 2380-2400. doi: 10.1093/mnras/stz590

Murphy, S. J., Moe, M., Kurtz, D. W., Bedding, T., Shibahashi, H., and Boffin, H. M. J. (2018a). VizieR Online Data Catalog: Orbital Parameters of 341 New Binaries (Murphy+ 2018). Strasbourg: VizieR Online Data Catalog.

Murphy, S. J., Moe, M., Kurtz, D. W., Bedding, T. R., Shibahashi, H., and Boffin, H. M. J. (2018b). Finding binaries from phase modulation of pulsating stars with Kepler: V. Orbital parameters, with eccentricity and mass-ratio distributions of 341 new binaries. Mon. Not. RAS 474, 4322-4346. doi: 10.1093/mnras/ stx3049

Murphy, S. J., and Paunzen, E. (2017). Gaia's view of the $\lambda$ Boo star puzzle. Mon. Not. RAS 466, 546-555. doi: 10.1093/mnras/stw3141

Murphy, S. J., Saio, H., Takada-Hidai, M., Kurtz, D. W., Shibahashi, H., Takata, M., et al. (2020). On the first $\delta$ Sct-roAp hybrid pulsator and the stability of $\mathrm{P}$ and G modes in chemically peculiar A/F stars. Mon. Not. RAS 498, 4272-4286. doi: $10.1093 / \mathrm{mnras} / \mathrm{staa} 2667$

Murphy, S. J., Shibahashi, H., and Kurtz, D. W. (2013). Super-Nyquist asteroseismology with the Kepler space telescope. Mon. Not. RAS 430:2986-2998. doi: 10.1093/mnras/stt105

Nemec, J. M., Balona, L. A., Murphy, S. J., Kinemuchi, K., and Jeon, Y.-B. (2017). Metal-rich SX Phe stars in the Kepler field. Mon. Not. RAS 466, 1290-1329. doi: $10.1093 / \mathrm{mnras} / \mathrm{stw} 3072$

Ouazzani, R.-M., Salmon, S. J. A. J., Antoci, V., Bedding, T. R., Murphy, S. J., and Roxburgh, I. W. (2017). A new asteroseismic diagnostic for internal rotation in $\gamma$ Doradus stars. Mon. Not. RAS 465, 2294-2309. doi: 10.1093/mnras/stw2717

Ouazzani, R. M., Lignières, F., Dupret, M. A., Salmon, S. J. A. J., Ballot, J., Christophe, S., et al. (2020). First evidence of inertial modes in $\gamma$ Doradus stars: the core rotation revealed. Astron. Astrophys. 640:A49. doi: 10.1051/0004-6361/201936653

Paparó, M., Benkő, J. M., Hareter, M., and Guzik, J. A. (2016a). Unexpected series of regular frequency spacing of $\delta$ Scuti stars in the non-asymptotic regime. I. The methodology. Astrophys. J. 822:100. doi: 10.3847/0004-637X/822/2/100

Paparó, M., Benkő, J. M., Hareter, M., and Guzik, J. A. (2016b). Unexpected series of regular frequency spacing of $\delta$ Scuti stars in the non-asymptotic regime. II. Sample-Echelle diagrams and rotation. Astrophys. J. Suppl. 224:41. doi: 10.3847/0067-0049/224/2/41

Pedersen, M. G., Antoci, V., Korhonen, H., White, T. R., Jessen-Hansen, J., Lehtinen, J., et al. (2017). Do A-type stars flare? Mon. Not. RAS 466, 3060-3076. doi: $10.1093 / \mathrm{mnras} / \mathrm{stw} 3226$ 
Pinsonneault, M. H., An, D., Molenda-Żakowicz, J., Chaplin, W. J., Metcalfe, T. S., and Bruntt, H. (2012). A revised effective temperature scale for the Kepler input catalog. Astrophys. J. Suppl. 199:30. doi: 10.1088/0067-0049/199/2/30

Pinsonneault, M. H., An, D., Molenda-Żakowicz, J., Chaplin, W. J., Metcalfe, T. S., and Bruntt, H. (2013). Erratum: "A Revised Effective Temperature Scale for the Kepler Input Catalog” (2012, ApJS 199:30). Astrophys. J. Suppl. 208:12. doi: 10.1088/0067-0049/208/1/12

Pollard, K. R. (2009). “A review of $\gamma$ Doradus variables," in Stellar Pulsation: Challenges for Theory and Observation, Volume 1170 of American Institute of Physics Conference Series, eds J. A. Guzik and P. A. Bradley (New York, NY: American Institute of Physics, Melville), 455-466.

Pollard, K. R., Brunsden, E. J., Cottrell, P. L., Davie, M. W., Greenwood, A., and Kilmartin, P. M. (2013). "Spectroscopic mode identification in $\gamma$ Doradus stars," in Progress in Physics of the Sun and Stars: A New Era in Helio- and Asteroseismology, Volume 479 of Astronomical Society of the Pacific Conference Series, eds H. Shibahashi and A. E. Lynas-Gray (San Francisco, CA), 105.

Poretti, E., Michel, E., Garrido, R., Lefèvre, L., Mantegazza, L., Rainer, M., et al. (2009). HD 50844: a new look at $\delta$ Scuti stars from CoRoT space photometry. Astron. Astrophys. 506, 85-93. doi: 10.1051/0004-6361/200912039

Rain, M. J., Ahumada, J., and Carraro, G. (2021). A new, Gaia based, catalogue of blue straggler stars in open clusters. arXiv 2103.06004. doi: 10.1051/0004-6361/202040072

Ricker, G. R., Winn, J. N., Vanderspek, R., Latham, D. W., Bakos, G. Á., Bean, J. L., et al. (2015). Transiting exoplanet survey satellite (TESS). J. Astron. Telesc. Instr. Syst. 1:014003. doi: 10.1117/1.JATIS.1.1.014003

Rodríguez, E., and Breger, M. (2001). Delta Scuti and related stars: analysis of the R00 catalogue. Astron. Astrophys. 366, 178-196. doi: 10.1051/0004-6361:20000205

Saio, H., Kurtz, D. W., Murphy, S. J., Antoci, V. L., and Lee, U. (2018). Theory and evidence of global Rossby waves in upper main-sequence stars: R-mode oscillations in many Kepler stars. Mon. Not. RAS 474, 2774-2786. doi: 10.1093/mnras/stx2962

Saio, H., Kurtz, D. W., Takata, M., Shibahashi, H., Murphy, S. J., Sekii, T., et al. (2015). Asteroseismic measurement of slow, nearly uniform surface-tocore rotation in the main-sequence F star KIC 9244992. Mon. Not. RAS 447, 3264-3277. doi: 10.1093/mnras/stu2696

Sandquist, E. L., Jessen-Hansen, J., Shetrone, M. D., Brogaard, K., Meibom, S., Leitner, M., et al. (2016). The age and distance of the Kepler open cluster NGC 6811 from an eclipsing binary, turnoff star pulsation, and giant asteroseismology. Astrophys. J. 831:11. doi: 10.3847/0004-637X/831/1/11

Schmid, V. S., and Aerts, C. (2016). Asteroseismic modelling of the two Ftype hybrid pulsators KIC 10080943A and KIC 10080943B. Astron. Astrophys. 592:A116. doi: 10.1051/0004-6361/201628617

Sikora, J., David-Uraz, A., Chowdhury, S., Bowman, D. M., Wade, G. A., Khalack, V., et al. (2019). MOBSTER-II. Identification of rotationally variable A stars observed with TESS in sectors 1-4. Mon. Not. RAS 487, 4695-4710. doi: $10.1093 / \mathrm{mnras} / \mathrm{stz} 1581$

Smalley, B., Antoci, V., Holdsworth, D. L., Kurtz, D. W., Murphy, S. J., De Cat, P., et al. (2017). Pulsation versus metallicism in Am stars as revealed by LAMOST and WASP. Mon. Not. RAS 465, 2662-2670. doi: 10.1093/mnras/stw2903

Suárez, J. C., García Hernández, A., Moya, A., Rodrigo, C., Solano, E., Garrido, R., et al. (2014). Measuring mean densities of $\delta$ Scuti stars with asteroseismology.
Theoretical properties of large separations using TOUCAN. Astron. Astrophys. 563:A7. doi: 10.1051/0004-6361/201322270

Tassoul, M. (1980). Asymptotic approximations for stellar nonradial pulsations. Astrophys. J. Suppl. 43, 469-490. doi: 10.1086/190678

Thompson, S. E., Everett, M., Mullally, F., Barclay, T., Howell, S. B., Still, M., et al. (2012). A class of eccentric binaries with dynamic tidal distortions discovered with Kepler. Astrophys. J. 753:86. doi: 10.1088/0004-637X/753/1/86

Tingley, B., Bonomo, A. S., and Deeg, H. J. (2011). Using stellar densities to evaluate transiting exoplanetary candidates. Astrophys. J. 726:112. doi: 10.1088/0004-637X/726/2/112

Uytterhoeven, K., Moya, A., Grigahcène, A., Guzik, J. A., Gutiérrez-Soto, J., Smalley, B., et al. (2011). The Kepler characterization of the variability among A- and F-type stars. I. General overview. Astron. Astrophys. 534:A125. doi: 10.1051/0004-6361/201117368

Van Reeth, T., Mombarg, J. S. G., Mathis, S., Tkachenko, A., Fuller, J., Bowman, D. M., et al. (2018). Sensitivity of gravito-inertial modes to differential rotation in intermediate-mass main-sequence stars. Astron. Astrophys. 618:A24. doi: 10.1051/0004-6361/201832718

Van Reeth, T., Tkachenko, A., and Aerts, C. (2016). Interior rotation of a sample of $\gamma$ Doradus stars from ensemble modelling of their gravity-mode period spacings. Astron. Astrophys. 593:A120. doi: 10.1051/0004-6361/201628616

Van Reeth, T., Tkachenko, A., Aerts, C., Pápics, P. I., Degroote, P., Debosscher, J., et al. (2015a). Detecting non-uniform period spacings in the Kepler photometry of $\gamma$ Doradus stars: methodology and case studies. Astron. Astrophys. 574:A17. doi: 10.1051/0004-6361/201424585

Van Reeth, T., Tkachenko, A., Aerts, C., Pápics, P. I., Triana, S. A., Zwintz, K., et al. (2015b). Gravity-mode period spacings as a seismic diagnostic for a sample of $\gamma$ Doradus stars from Kepler space photometry and high-resolution ground-based spectroscopy. Astrophys. J. Suppl. 218:27. doi: 10.1088/0067-0049/218/2/27

Welsh, W. F., Orosz, J. A., Aerts, C., Brown, T. M., Brugamyer, E., Cochran, W D., et al. (2011). KOI-54: The Kepler discovery of tidally excited pulsations and brightenings in a highly eccentric binary. Astrophys. J. Suppl. 197:4. doi: 10.1088/0067-0049/197/1/4

White, T. R., Pope, B. J. S., Antoci, V., Pápics, P. I., Aerts, C., Gies, D. R., et al. (2017). Beyond the Kepler/K2 bright limit: variability in the seven brightest members of the Pleiades. Mon. Not. RAS 471, 2882-2901. doi: $10.1093 / \mathrm{mnras} / \mathrm{stx} 1050$

Xiong, D. R., Deng, L., Zhang, C., and Wang, K. (2016). Turbulent convection and pulsation stability of stars-II. Theoretical instability strip for $\delta$ Scuti and $\gamma$ Doradus stars. Mon. Not. RAS 457, 3163-3177. doi: 10.1093/mnras/stw047

Conflict of Interest: The author declares that the research was conducted in the absence of any commercial or financial relationships that could be construed as a potential conflict of interest.

Copyright (c) 2021 Guzik. This is an open-access article distributed under the terms of the Creative Commons Attribution License (CC BY). The use, distribution or reproduction in other forums is permitted, provided the original author(s) and the copyright owner(s) are credited and that the original publication in this journal is cited, in accordance with accepted academic practice. No use, distribution or reproduction is permitted which does not comply with these terms. 